\title{
Norois
}

Environnement, aménagement, société

194 | 2005/1

Les milieux polaires et subpolaires de l'Atlantique

\section{Chronique polaire}

Samuel Étienne, Denis Mercier et Marie-Françoise André

\section{(2) OpenEdition}

Journals

Édition électronique

URL : http://journals.openedition.org/norois/698

DOI : $10.4000 /$ norois. 698

ISBN : 978-2-7535-1542-0

ISSN : 1760-8546

Éditeur

Presses universitaires de Rennes

Édition imprimée

Date de publication : 1 mars 2005

Pagination : 125-148

ISBN : 978-2-7535-0100-3

ISSN : 0029-182X

Référence électronique

Samuel Étienne, Denis Mercier et Marie-Françoise André, « Chronique polaire », Norois [En ligne], 194 | 2005/1, mis en ligne le 28 avril 2009, consulté le 19 avril 2019. URL : http:// journals.openedition.org/norois/698; DOI : 10.4000/norois.698

Ce document a été généré automatiquement le 19 avril 2019.

(c) Tous droits réservés 


\title{
Chronique polaire
}

\author{
Samuel Étienne, Denis Mercier et Marie-Françoise André
}

À la mémoire de Max Derruau

Depuis 1954, les lecteurs fidèles de Norois ont régulièrement rendez-vous avec la chronique des hautes latitudes arctiques initiée et alimentée pendant dix ans par CharlesPierre Péguy, Jean Corbel et Pierre Biays. À leur suite, Alice Saunier-Picard publiera une douzaine de chroniques de 1966 à 1973. Annick Moign et Jean-Claude Bodéré lui succéderont jusqu'en 1984, épaulés à partir de 1982 par François Carré et Thierry Simon. Pendant plus de dix ans, après la disparition tragique d'Annick Moign, la chronique s'effacera des pages de Norois, puis, en 1997, Marie-Françoise André reprendra le flambeau pour une chronique qui s'élargira au continent antarctique. Aujourd'hui, à l'invitation de cette dernière, c'est une équipe plus étoffée qui tentera de rendre compte de l'actualité scientifique (et parfois même, extra-scientifique) des régions polaires, s'inscrivant dans une tradition de recension commentée et initiée il y a un siècle par le géographe Charles Rabot qui publiait des chroniques, comptes rendus d'articles et projet d'exploration polaire dans $L a$ Géographie $^{1}$. La mémoire de Charles Rabot était d'ailleurs récemment à l'honneur puisque le 8 septembre 2001, Son Excellence Monsieur Louis Amigues, ambassadeur de France en Norvège, inaugurait la deuxième base scientifique française en Arctique, à Ny-Ålesund, au Spitsberg. Cette base porte donc le nom de ce géographe, hommage à celui qui fut l'un des précurseurs de la coopération scientifique franconorvégienne et de la recherche polaire française au Spitsberg. Elle vient renforcer la présence française en presqu'île de Brøgger où, depuis 1963, fonctionne la base Jean Corbel (fig. 1) dont la rénovation fut saluée le même jour par la délégation protocolaire. Deux bases arctiques portant les noms d'illustres géographes, voilà un bel hommage à la présence, ancienne et continue, des géographes français dans les hautes latitudes de l'hémisphère boréale ${ }^{2}$. 
Figure 1 : Base française « Jean Corbel » en baie du Roi, Spitsberg, $79^{\circ} \mathrm{N}$ French camp "Jean Corbel" in the Kongsford, Spitsbergen, $79^{\circ} \mathrm{N}$

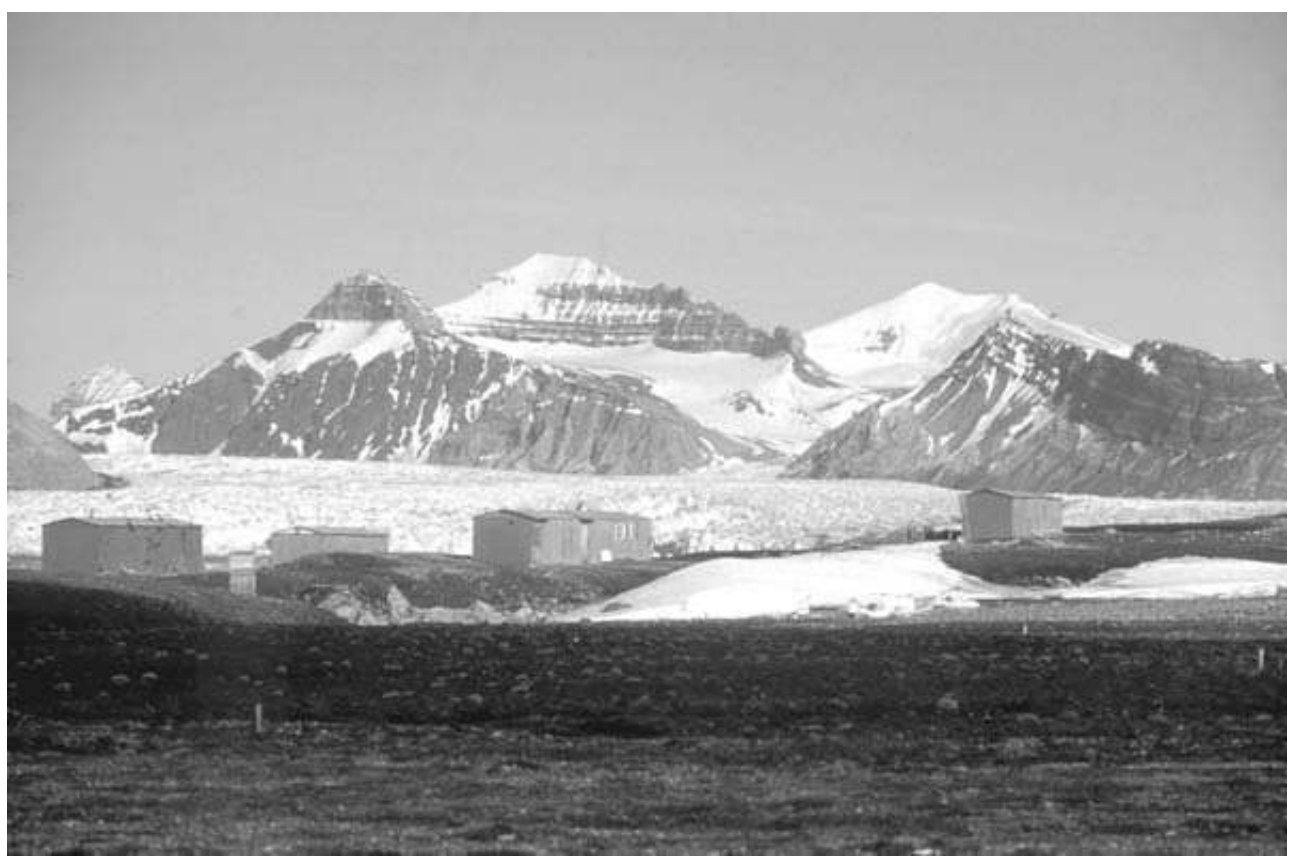

(ㄱ) M.-F. André)

\section{Tourisme polaire}

\section{Antarctique et sub-antarctique}

2 Phénomène social récent en Antarctique, le tourisme n'avait fait l'objet, jusque dans les années 2000, que de recherches ponctuelles sur la fréquentation, les caractéristiques sociologiques et les impacts environnementaux des touristes. Un programme de recherche plus systématique a été lancé récemment par des équipes néo-zélandaises ${ }^{3}$ dont le triple objectif est d'approfondir la connaissance de l'« expérience antarctique » avant, pendant et après le séjour touristique. Le postulat initial de ces chercheurs est que les touristes en Antarctique revendiquent plus qu'ailleurs l'adoption de valeurs écotouristiques (information, conservation, protection...). L'étude est donc déclinée en trois temps : un examen pré-voyage des motivations des touristes (via un questionnaire diffusé par courrier postal), un suivi in situ durant le séjour (entretiens longs lors des liaisons maritimes, observation des comportements sur site afin de déceler d'éventuels décalages entre discours et pratiques), et un bilan ultérieur (par courrier électronique deux à six mois après le retour) des profits tirés de l'expérience du voyage sur le continent antarctique. Les résultats préliminaires de l'étude laissent apparaitre que l'expérience touristique en Antarctique est autant bénéfique à l'individu lui-même qu'aux lieux visités puisqu'elle augmente la sensibilité de celui-là à la nécessité de protéger ces milieux sensibles.

Les îles Shetland du Sud sont la principale zone de fréquentation touristique de la péninsule antarctique avec une croissance de plus de $500 \%$ du nombre de touristes depuis 15 ans (supérieur à 10000 depuis l'hiver 1998-1999). Cet archipel doit son 
exceptionnel tropisme à sa diversité paysagère (paysages glaciaires, volcaniques, littoraux), sa richesse faunistique typiquement antarctique (colonies de manchots, d'éléphants de mer, etc., fig. 2), mais également à un patrimoine historique qui, bien que modeste, véhicule une forte charge émotionnelle : la visite de la plaque commémorative du dernier campement de l'expédition Shackleton sur lîle Éléphant constitue ainsi « une introduction à l'ambiance antarctique maritime pour les routes touristiques ${ }^{4} »$. Cette fréquentation touristique en hausse, concentrée autour de quelques points de débarquement, pose déjà le problème de la conservation des sites et de la gestion d'une pression anthropique croissante et de plus en plus visible (abandon de déchets, perturbation des animaux, pollution mineure des eaux lors des opérations de débarquement). Enrique Serrano Cañadas fait le point sur le potentiel touristique de cet archipel, son utilisation actuelle et les perspectives de développement dans le cadre du Traité de l'Antarctique.

Figure 2 : Colonie de manchots empereurs (Aptenodytes forsteri) à Kerguelen Emperor penguins (Aptenodytes forsteri) rookerie at Kerguelen island

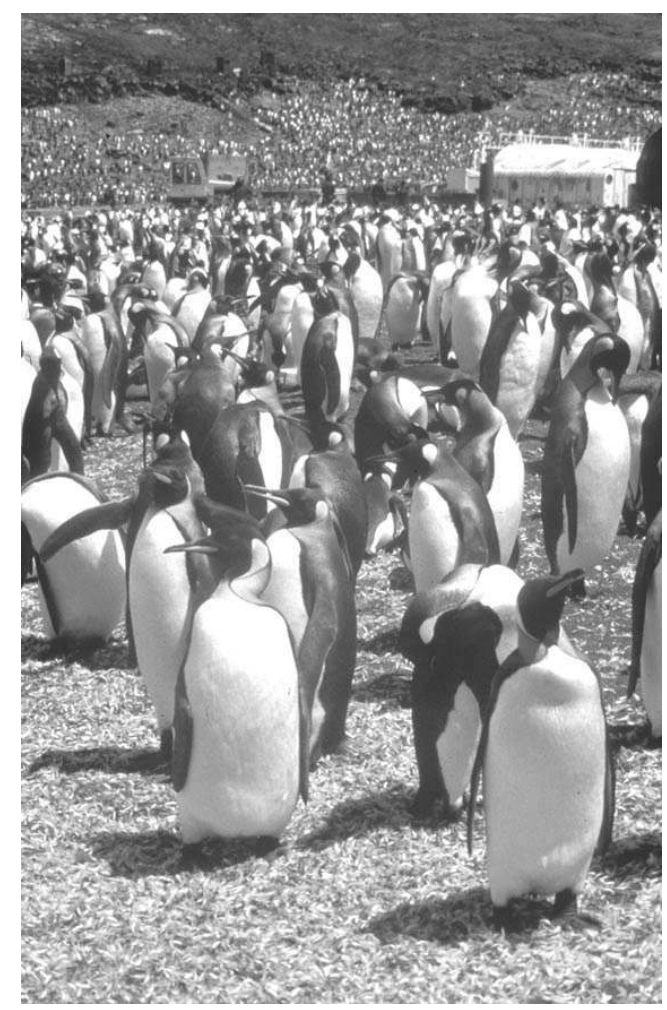

( B. Labidoire)

Inclus dans la même dynamique régionale que le continent antarctique et suivant une tendance similaire mais à un degré supérieur, le tourisme aux îles Malouines a connu une progression tout aussi vertigineuse dans la seconde moitié des années 1990, passant de moins de 5000 visiteurs en 1994-1995 à près de 38000 en 1999-20005. Cette évolution est due autant à l'augmentation de la fréquence des visites qu'à celle du gabarit des bateaux de croisière, et notamment l'arrivée des bateaux grand luxe emportant plus de 1000 passagers. Inévitablement, ceci induit une multiplication des sites terrestres visités, une pression grandissante et plus diffuse sur la flore et la faune alors qu'aucun arsenal législatif ne régule le tourisme sur l'archipel. La question de la gestion du tourisme se 
pose d'autant plus que les bateaux à grande capacité ne peuvent être affiliés à l' International Association of Antarctica Tour Operators (IAATO) et n'ont donc aucune obligation d'en suivre les standards environnementaux. Des prévisions faisant état d'une fréquentation atteignant très vite les 50000 visiteurs annuels invitent les autorités locales à adopter urgemment une politique touristique raisonnée et à mettre en place un code de conduite pour l'ensemble des tour-operateurs.

Les effets écologiques du piétinement font désormais l'objet d'un suivi plus systématique ${ }^{6}$ : sur l'île Marion, il apparaît, sans grande surprise, que la nature des formations végétales (toundras humide, herbacée ou pierreuse) combinée aux conditions de sites (pente, nature et structure des sols) déterminent la sensibilité des lieux au piétinement. Si la tendance générale est une réponse négative au piétinement (dont témoigne l'élargissement des chemins de passage), les formations sèches des versants se montrent beaucoup moins affectées que les étendues marécageuses des fonds de vallée. En revanche, les compositions spécifiques des formations végétales montrent un bouleversement plus net à travers l'appauvrissement de la diversité spécifique (au détriment des fougères et des plantes ligneuses, mais au bénéfice des graminées et des espèces introduites).

\section{Arctique et sub-arctique}

6 La mise en tourisme de la région arctique est beaucoup plus ancienne que celle de l'aire polaire australe mais elle présente toutefois une hétérogénéité tant chronologique que structurelle suivant les pays : si le Groenland a suscité l'intérêt des touristes européens dès le début du vingtième siècle - dès 1902, l'explorateur danois Mylius Erichsen voulut y organiser deux croisières estivales pour une centaine de touristes anglais mais ne reçut pas les autorisations nécessaires -, ce n'est qu'à partir de 1959 que des vols réguliers depuis le Danemark ou l'Islande vont assurer une activité régulière bien que modeste (3 500 visiteurs recensés en 1992) 7 . En 1991, le Gouvernement groenlandais proposa de faire du tourisme l'un des trois axes majeurs de développement économique du pays en se fixant pour objectif 35000 visiteurs à l'horizon 2005. Ce niveau de fréquentation touristique devrait permettre la création de 2 200-2 500 emplois directs permanents, 1000 emplois indirects et rapporter près de 500M DKK (environ 67M EUR). En 1997, la fréquentation atteignait déjà 17000 visiteurs et près de 32000 touristes avait séjourné dans le pays en 2000. Toutefois, il apparait que les conséquences environnementales et sociétales de l'activité touristique au Groenland n'ont fait l'objet ni de recherches préalables ni de suivi, posant le problème de la pérennité d'un secteur qui repose, pour beaucoup, sur la quête de l'« expérience sauvage ».

7 La faune polaire, terrestre ou maritime, constitue l'un des principaux facteurs d'attraction des milieux froids (fig. 3). Son exploitation repose sur différents types de produits touristiques comme l'observation à distance (watching), le safari photographique ou même la chasse. La forte croissance du secteur touristique provoque la multiplication des contacts homme-animal et conduit inévitablement à une altération des conditions de vie sauvage. Des études de plus en plus nombreuses se penchent donc sur les interactions entre tourisme et vie sauvage afin de définir des règles d'exploitation de la faune polaire ne mettant en péril ni l'animal ni l'activité économique sur le long terme. À Churchill (Manitoba, Canada), l'observation des ours polaires à bord de véhicules tout-terrain grillagés est pratiquée en octobre-novembre de chaque année ( 45 jours en moyenne) 
depuis les années 1980. Durant cette période, les ours se concentrent autour de Gordon Point dans l'attente de la reprise en glace de la baie d'Hudson qu'ils ont dû quitter durant l'été et la fonte de la banquise. Cette période est critique d'un point de vue physiologique puisque ces animaux s'y nourrissent mal et passent le plus clair de leur temps au repos afin de ne pas puiser dans leur réserve graisseuse. Une étude de Dycka et Baydack ${ }^{8}$ montre que l'observation depuis les véhicules motorisés des ours polaires dans cette région (bear-watching) perturbe le comportement des ours: elle provoque un accroissement de la vigilance (relèvement de la tête et observation des alentours) et une augmentation de l'activité métabolique chez les mâles semblables à l'apparition d'un congénère, seul danger potentiel pour un animal qui n'a pas de prédateur naturel. Au contraire, les femelles accompagnées d'oursons se montrent plus tranquilles en présence des véhicules qui semblent "perçus » comme un répulsif à ours mâles, danger potentiel majeur pour leur progéniture.

Figure 3 : L'observation de la faune sauvage est l'une des premières motivations du tourisme polaire, ici un phoque barbu (Erignathus barbatus) en Baie du Roi, Spitsberg, $79^{\circ} \mathrm{N}$ Wildlife-watching is one of most attractive activities in polar tourism, here a bearded seal (Erignathus barbatus) in the Kongsford area, Spitsbergen, $79^{\circ} \mathrm{N}$

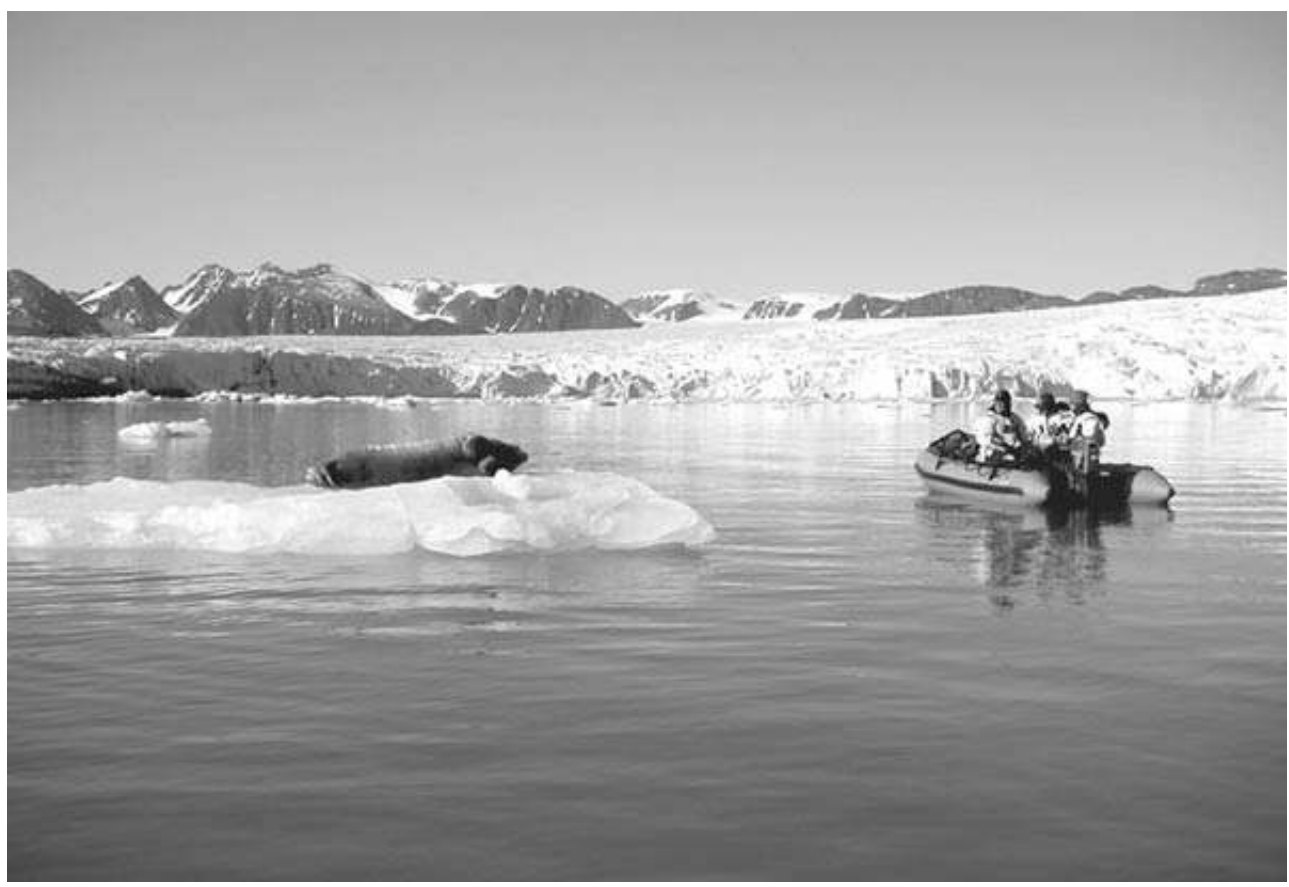

(@ S. Étienne)

8 Être en contact avec une nature vierge, observer des animaux sauvages dans leur environnement naturel sont autant d'atouts pour le tourisme polaire que des sources de préoccupation pour les autorités locales en charge de la protection de l'environnement. Le tourisme polaire, s'il est une force de développement économique incontournable, suscite de nombreuses craintes quant à la capacité d'absorption de ces régions de flux de touristes à fort taux de croissance. Les stratégies des gestionnaires diffèrent fortement en Arctique : des mesures de plus en plus restrictives sont appliquées au Svalbard, alors que le Groenland a largement privilégié des mesures incitatives. Ces stratégies s'inscrivent dans un cadre plus général de réflexion sur les politiques de développement touristique en Arctique?. 
9 La pregnance des composantes naturelles au sein des paysages polaires et subpolaires octroie aux produits touristiques de ces milieux un fort potentiel écotouristique. Toutefois, à y regarder de plus près, on peut se poser la question de l'usage qui est fait de ce potentiel et si derrière l'alibi écotouristique ne se cache pas des activités plus proches de l'éco-exploitation que de la conciliation protection de l'environnement/valorisation touristique ? Magali Chauveau a ainsi analysé, sous l'angle de la mercatique, les produits touristiques offerts sur le territoire islandais et tenté de mesurer leur «valeur écotouristique ${ }^{10} »$. Ainsi, en termes quantitatifs et qualitatifs, la majorité des produits touristiques offerts sur le territoire islandais ne répondent pas aux critères les plus stricts de l'écotourisme.

\section{L'hydrologie polaire}

La montée en puissance des recherches en relation avec les changements climatiques explique certainement en partie le nouvel essor des recherches hydrologiques dans les milieux polaires. Le suivi hydrologique des bassins versants englacés permet de résoudre partiellement la question de base sur l'apport de la fonte des glaciers polaires dans l'élévation du niveau de la mer observé au cours $d u x^{e}$ siècle en relation avec les changements climatiques.

11 Un colloque à Ny-Ålesund a été organisé en 2003 par deux collègues espagnols, Adolpho Eraso et Carmen Domínguez, et s'est tenu dans le cadre de la commission internationale "Glacier caves and karst in Polar Regions (CLACKIPR) ", après les rencontres de Madrid (1990), Silésie (1992), Chamonix (1994), Rudolfschütte (1996) et Courmayeur (2000). Les actes rapidement publiés ${ }^{11}$ apportent des éléments chiffrés très précieux dont voici les principaux enseignements.

12 Les lecteurs assidus de Norois connaissent bien la presqu'île de Brøgger au Spitsberg nordoccidental. Les travaux d'Adolpho Eraso et Carmen Domínguez sur le bassin versant de l'Austre Lovénbreen, proche de la base française Jean-Corbel, sont abondamment développés dans ces actes. Ils s'inscrivent dans la longue histoire des recherches hydrologiques menées précédemment par les Français dans les années 1960 avec H. Geoffray et H. Vivian, puis dans les années 1980 avec les travaux de M. Griselin. Depuis 2001, A. Eraso et C. Domínguez ont équipé le bassin versant de l'Austre Lovénbreen (fig. 4) et apportent de précieuses informations d'une grande précision (tableau 1). L'écoulement annuel ne dure que 77 jours par an en 2001 et 2002, valeur déjà proposée par H. Geoffray dans sa thèse en 1968. Ils montrent une parfaite corrélation négative entre la conductivité et le débit $\left(C=136,1 D^{-0,5523}\right.$ avec $\left.R^{2}=0,97\right)$. Ceci prouve que plus le débit est grand moins les eaux ont de temps de contact avec le substratum et par conséquent que les rivières drainent très rapidement leur bassin versant. La conductivité des eaux provient ici de l'hydrolyse des roches cristallines, majoritairement représentées, et de la dissolution des roches carbonatées du bassin versant de l'Austre Lovénbreen. Les mêmes auteurs arrivent à la conclusion inverse dans la relation entre débit et conductivité pour le bassin versant du Bellingshausen Dome sur l'île du Roi George dans les Shetlands du Sud (62 $11^{\prime}$ ' $; 58^{\circ}$ $54^{\prime}$ W). L'existence d'une zone saturée en arrière d'une moraine frontale explique cette différence. L'écoulement de cette rivière ne s'est effectué que durant 87 jours en 2002 avec un débit spécifique de $0,486 \mathrm{~m}^{3} \cdot \mathrm{s}^{-1} \cdot \mathrm{km}^{-2}$ entre le 20 janvier 2002 et le 31 décembre de la même année. 
Figure 4 : Le bassin versant de l'Austre Lovénbreen le 9 juillet 2004

Austre Lovénbreen catchment, 2004 July 9

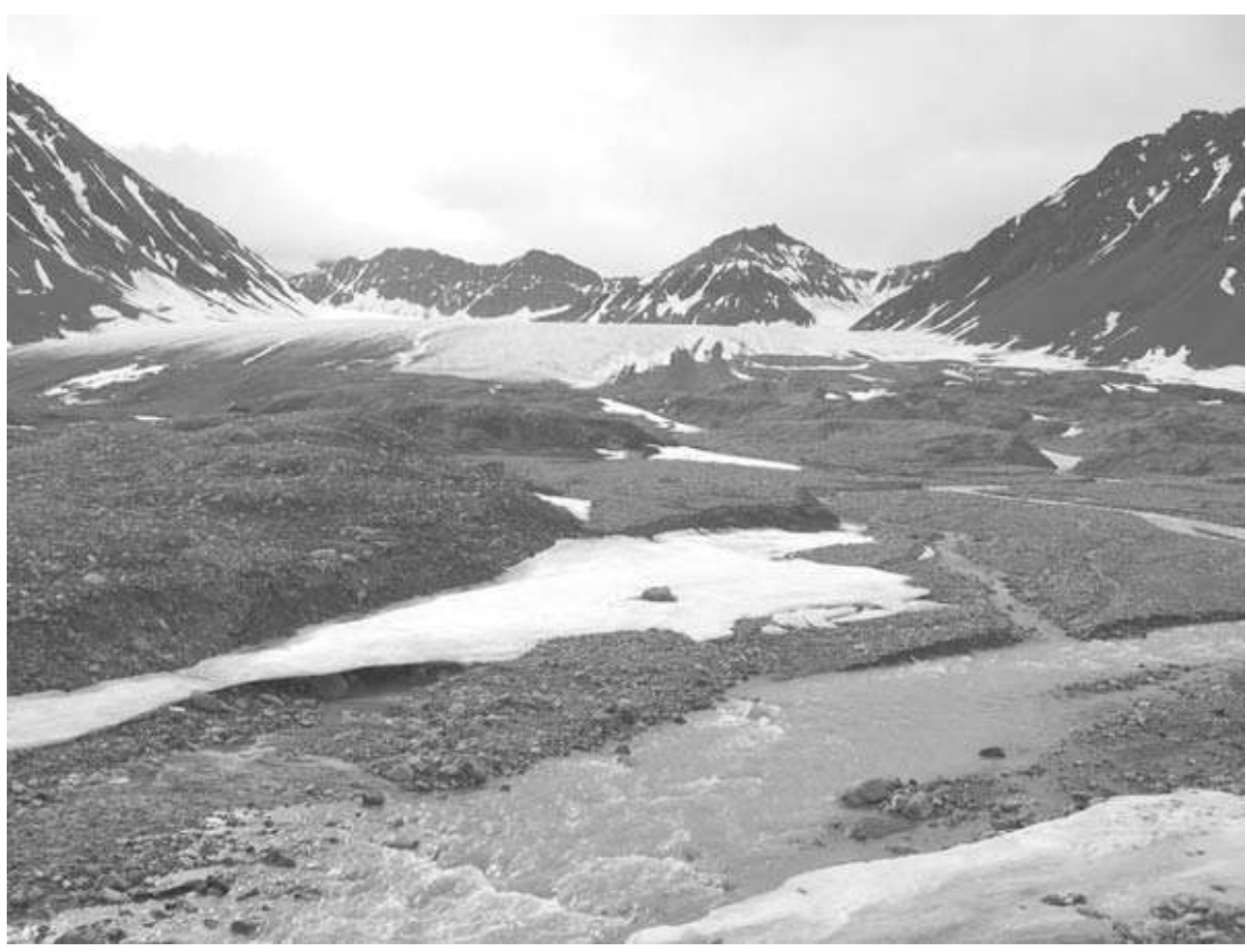

( D. Mercier)

Tableau 1 : Données hydrologiques de bassins versants polaires (d'après. A. Eraso et $\mathrm{C}$. Domínguez, 2004)

Hydological datas from polar catchments (in A. Eraso and C. Domínguez, 2004)

\begin{tabular}{|c|c|c|c|c|c|c|c|}
\hline \multirow[b]{2}{*}{ nom de code } & \multicolumn{2}{|c|}{$\begin{array}{c}\text { Antarctique } \\
\left(62^{\circ} \mathrm{S}\right)\end{array}$} & \multicolumn{3}{|c|}{$\begin{array}{c}\text { Svalbard } \\
\left(79^{\circ} \mathrm{N}\right)\end{array}$} & \multicolumn{2}{|c|}{$\begin{array}{c}\text { Islande } \\
\left(64-66^{\circ} \mathrm{N}\right)\end{array}$} \\
\hline & CPE-SC-62\% & CPE-BCAA-62'S & & E-ALB-79 & & CPE-KVIA-64N & CPE-KALD-66'N \\
\hline surface $\left(\mathrm{km}^{2}\right)$ & 1,125 & 1,3 & & 6,28 & & 13 & 49,5 \\
\hline LEG (m) & 270 & 270 & & 300 & & 1050 & 650 \\
\hline année & 2000 & 2002 & 2001 & 2002 & 2003 & 2000 & 2000 \\
\hline $\begin{array}{l}\text { débit brut } \\
\left(\mathrm{m}^{3} \cdot \mathrm{s}^{-1}\right)\end{array}$ & 0,33 & 0,50 & 1,69 & 1,83 & 2,68 & 22,00 & 34,66 \\
\hline $\begin{array}{l}\text { débit spécifique } \\
\left(\mathrm{m}^{3} \cdot \mathrm{s}^{-1} \cdot \mathrm{km}^{-2}\right)\end{array}$ & 0,29 & 0,38 & 0,27 & 0,29 & 0,43 & 1,69 & 0,70 \\
\hline
\end{tabular}

13 Les analyses statistiques d'Adolpho Eraso et Carmen Domínguez à partir des données hydrologiques de l'Austre Lovénbreen apportent des éléments de réflexion fondamentaux pour les recherches hydrologiques futures. En comparant les débits des séries prises toutes les 5 minutes, une fois par heure, toutes les 4 heures, toutes les 8 heures et une fois par jour, les résultats statistiques montrent, à partir des moyennes, des minimums et des maximums, que l'idéal d'un point de vue statistique est d'avoir une mesure toutes les 4 heures. Ceci permet de suivre au plus près l'évolution des débits, sans perdre les 
variations intra-journalières, importantes dans ces milieux, sans non plus dépasser trop rapidement les capacités de stockage des mémoires des appareils de mesure. Il serait en effet délicat, après ces résultats d'une grande précision statistique, de ne proposer qu'une mesure journalière tant les écarts sont importants.

Les travaux de E. V. Chevnina et $\mathrm{O}$. N. Medkova ${ }^{12}$ sur les rivières Grendalen et Aldegonda dans le Grenfjord près de Barentsburg, toujours au Spitsberg, présentent à la fois les résultats des mesures hydrologiques menées depuis 2001 et l'évolution des glaciers depuis trente ans. Le bassin versant de la rivière Grendalen représente une superficie de $95 \mathrm{~km}^{2}$ qui n'est que partiellement englacé $(10 \%)$ ce qui explique que l'essentiel du $1,14 \mathrm{~m}^{3} \cdot \mathrm{s}^{-1}$ soit alimenté par la fonte nivale et par les eaux interstitielles émanant des zones marécageuses. La première source apporte des eaux boueuses à forte concentration minérale, tandis que la seconde source livre des eaux claires faiblement minéralisées. En revanche, les glaciers occupent $60 \%$ de la surface du bassin versant de la rivière Aldegonda qui s'élève à $20 \mathrm{~km}^{2}$. Ils contribuent presque seuls à l'alimentation de la rivière, les précipitations liquides ne représentant pas plus de 5 à $7 \%$ du débit global. L'évolution des débits est par conséquent logiquement corrélée avec celle de la

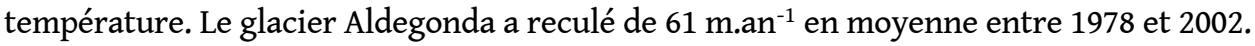

R. Mavluydov et I. Y. Solovyanova ${ }^{13}$ ont travaillé sur le glacier Aldegonda sur la côte ouest du Grønfjord, à $10 \mathrm{~km}$ au sud-ouest de Barentsburg au Spitsberg. Le front du glacier a reculé entre 1990 et 2002 de manière non uniforme de 200 à $450 \mathrm{~m}$ en fonction des différences d'épaisseur de la langue glaciaire. Entre 1985 et 2002 les débits sont passés de 80 litres par seconde à 1500 litres par seconde.

Le travail de Martin Gude ${ }^{14}$ sur les rythmes et les transports sédimentaires en milieu fluvial dans des petits bassins versants au Spitsberg s'inscrit dans cette thématique d'hydrologie polaire. Martin Gude offre une synthèse fort intéressante sur les débits, les transports dissous et solides au sein de trois petits bassins versants $\left(5 \mathrm{~km}^{2}\right)$, taillés dans des terrains sédimentaires et partiellement englacés, dans le Liefdefjorden au nord du Spitsberg $\left(79^{\circ} 33^{\prime} \mathrm{N}, 13^{\circ} \mathrm{E}\right)$. Il en ressort que les bassins versants partiellement occupés de glaciers polythermiques enregistrent des événements de grande ampleur érosive par rapport aux glaciers à base froide. Les débits liquides sont fortement corrélés aux conditions atmosphériques puisque la balance énergétique conditionne la fonte nivale, celle de la glace des glaciers et celle du pergélisol. Les petits bassins versants possèdent une faible capacité de rétention en raison de la réduction des infiltrations dans les sols gelés. Les rivières répondent de ce fait de manière rapide aux changements thermiques, qu'ils soient saisonniers ou diurnes (en relation avec les changements dans l'insolation $\mathrm{du}$ bassin versant). Ainsi, les variations de débits journaliers sont de l'ordre de $10001 . \mathrm{s}^{-1} \mathrm{et}$ les variations saisonnières de l'ordre de $2500 \mathrm{l.s}^{-1}$. En revanche, les événements extrêmes enregistrés s'élèvent à $120000001 . \mathrm{s}^{-1}$. Au début de la saison d'écoulement, les transports en solution sont perturbés par environ $20 \%$ de $\mathrm{Na}^{+}$et $\mathrm{Cl}^{-}$, ions déposés dans la neige. Ensuite, la part de $\mathrm{Ca}^{2+}$ et de $\mathrm{HCO}^{3-}$ s'accroît prouvant ainsi la contribution de la couche active et montrant la relation inverse entre la concentration et les débits. Les transports solides (en suspension et de fond) ne sont significatifs que dans les bassins versants possédant des glaciers polythermiques et absents des glaciers à base froide. Comparativement aux transports en solution (30\%) et suspension (69\%), les transports de fond demeurent négligeables (environ $1 \%$ ) selon l'auteur, même si des problèmes méthodologiques subsistent pour quantifier ces derniers. L'érosion varie ainsi de $41 \mathrm{t} . \mathrm{km}$ ${ }^{-2} \cdot \mathrm{a}^{-1}$ à $505 \mathrm{t} \cdot \mathrm{km}^{-2} \cdot \mathrm{a}^{-1}$, selon les bassins versants et les années de mesure. 

fluviale et l'érosion en milieu polaire et viennent enrichir une connaissance trop souvent ponctuelle dans le temps (quelques années de mesure seulement) et dans l'espace (de petits bassins versants dans l'immensité arctique). connaissancer organiques (mousses, insectes, spores, graines ou macro fossiles...), il a été démontré que le Longyearbreen, glacier de la vallée de Longyearbyen, capitale de l'archipel du Svalbard, était en retrait par rapport à sa position actuelle au moins entre 1880 et 1330 BP (calibré). Des recherches sont menées sur d'autres glaciers dans l'espoir de préciser la chronologie de cette partie de l'Holocène.

19 Un numéro spécial de Polar Research ${ }^{16}$ a été consacré aux recherches actuelles au Svalbard dans les différents domaines de la glaciologie, de l'érosion, du pergélisol, de la couverture neigeuse, de la botanique, de l'océanographie, de la biologie.

Par ailleurs, notons les travaux de Y. Y. Macheret et M. Y. Moskalevsky ${ }^{17}$ qui montrent le recul du Lange glacier d'un kilomètre depuis 1956 avec un réchauffement climatique de $1,3{ }^{\circ} \mathrm{C}$ dans le nord de la péninsule antarctique depuis les quatre dernières décennies.

21 Dans le domaine du cryokarst et de l'hydrologie glaciaire, la seconde édition de Cuevas en hielo y ríos bajo los glaciares s'est enrichie des dernières expéditions en milieu polaire ou subpolaire de ses auteurs, Adolfo Eraso et Marian Pulina, des universités de Madrid et de Silésie respectivement ${ }^{18}$. Les études menées en Antarctique, au Spitsberg, en Islande ou en Patagonie confirment le caractère anisotrope de la glace dans la zone d'ablation, propriété suggérée dans la première édition de l'ouvrage (1994). De nombreuses autres observations originales apportent de l'eau au moulin de la connaissance de la dynamique hydrogéologique des glaciers. En Islande par exemple, les auteurs ont remarqué que le barycentre d'une population de moulins restait immobile d'un point de vue géodésique malgré la déliquescence de la langue glaciaire (Kviarjökull). Le chapitre le plus notable reste celui consacré à la réponse, très rapide, des glaciers subpolaires au changement global et à la quantification de l'ablation glaciaire interne (ou ablation liquide), laquelle commence à être considérée comme le premier facteur d'élévation du niveau de la mer. À partir de différents exemples nord-atlantiques, antarctiques et subantarctiques, les auteurs remarquent la relation inverse entre l'ablation liquide et la latitude (en Islande, diminution de $31 \%$ avec une augmentation de deux degrés de latitude); la tendance à l'augmentation de celle-ci dans les deux hémisphères; l'accentuation du phénomène dans l'hémisphère boréal (à latitudes égales, l'ablation liquide y est quatre fois plus importante); et le fait que durant l'été austral, l'ablation liquide libèrerait 1000 fois plus d'eau liquide que le vêlage des icebergs depuis la calotte antarctique. Bien que ces observations apparaissent fondamentales, on remarquera avec regret que les conclusions des auteurs sont un peu hâtives puisque ne reposant, parfois, que sur deux séries annuelles de données, ce qui est loin d'être un minimum pour établir des tendances crédibles. Toutefois, l'instrumentation croissante de nombreux appareils glaciaires augure de grandes avancées dans la connaissance future du fonctionnement hydrogéologique des glaciers subpolaires dans un contexte de changement climatique. 


\section{Anthropisation et biodiversité dans les îles subantarctiques}

Les archipels de Kerguelen, Crozet et Amsterdam sont célèbres pour l'originalité de leur flore et de leur faune (fig. 5) qui présentent un taux d'endémicité élevé en raison de l'isolement de ces chapelets d'îles perdues dans l'océan Indien. Un numéro récent de la revue Pour la Science a été consacré à ces îles australes, véritables «laboratoires de l'évolution » dont la biodiversité est aujourd'hui menacée par des espèces végétales et animales introduites par l'homme depuis deux siècles ${ }^{19,20,21}$. Dès le XIX ${ }^{\mathrm{e}}$ siècle, phoquiers et baleiniers opérant dans les mers australes ont introduit - volontairement ou non - de nombreux mammifères dont sept sont encore présents à Kerguelen et/ou Crozet : le chat, le lapin, le mouton, le renne, le mouflon, la souris et le rat noir. Au début du $\mathrm{xx}^{\mathrm{e}}$ siècle, lors de l'installation de la ferme de Port-Couvreux, 1600 moutons importés des Malouines ont été installés à Kerguelen et après l'abandon de cet élevage, de nombreuses graminées introduites avec le fourrage de ces ovins se sont développées autour des bâtiments en ruine. Dans les années 1950, des moutons et mouflons ont été introduits et, vingt ans plus tard, des graminées fourragères ont été semées sur quatre îles de l'archipel de Kerguelen afin d'accroître les ressources alimentaires de ces animaux. Les années 1950-1960 ont également vu l'établissement de bases permanentes à Kerguelen, qui est à l'origine de l'introduction de nombreuses espèces végétales tempérées comme le pissenlit (Taraxacum officinale) et le pâturin annuel (Poa annua). S'y ajoutent des espèces nitrophiles qui se sont développées à proximité des rejets d'eaux usées, par exemple aux abords de la base Alfred-Faure à Crozet.

Figure 5 : Jeunes albatros à sourcils noirs (Diomedea melanophris), Kerguelen Young black-browed albatrosses (Diomedea melanophris) on Kerguelen

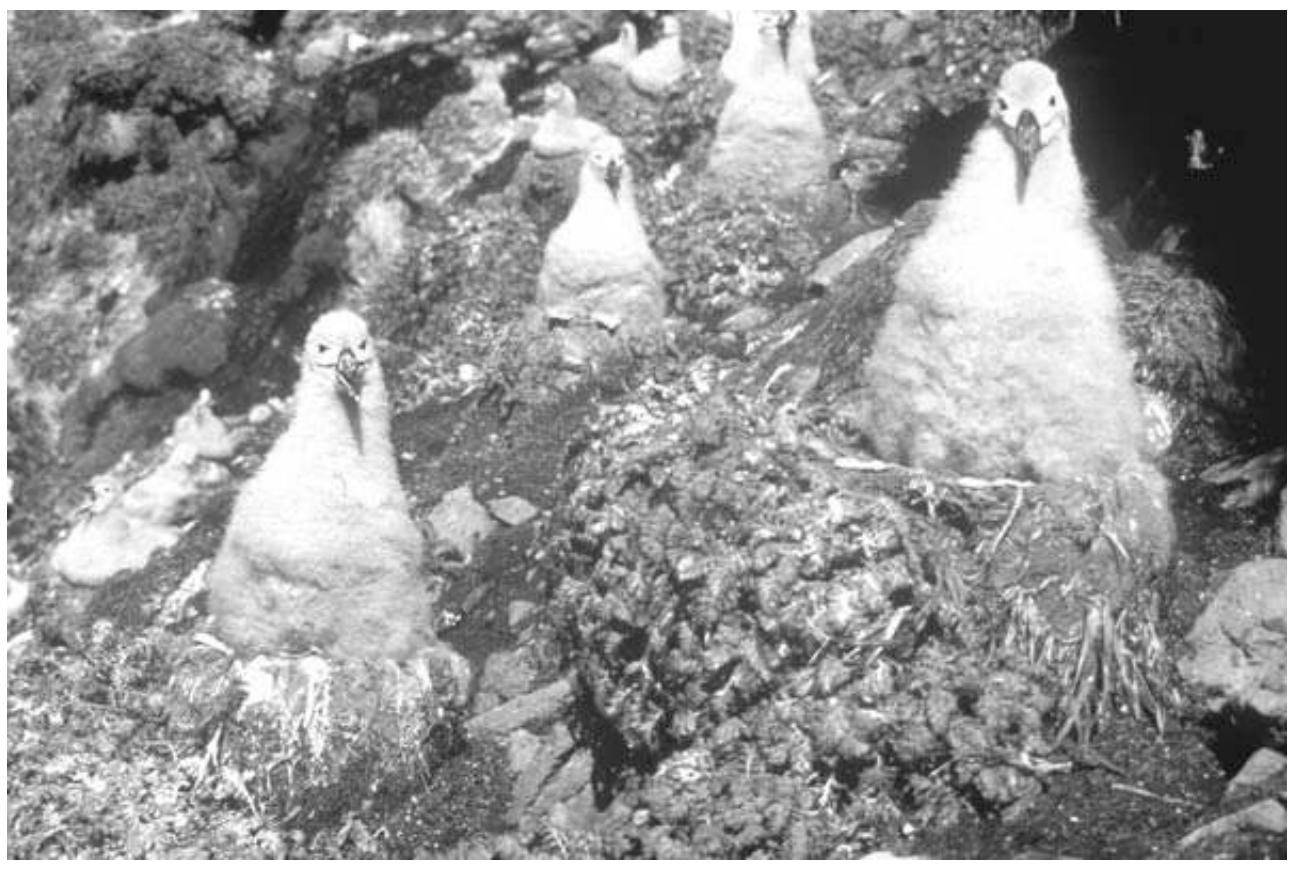

(๔ B. Labidoire) 
L'impact de ces espèces invasives a pu être évalué grâce à l'existence de zones-témoins indemnes d'introductions. S'agissant des plantes à fleurs, il apparaît que les espèces introduites restent pour la plupart strictement localisées et ne constituent pas pour l'instant une menace réelle pour la flore locale. Les plantes importées de la zone tempérée se trouvent ici dans des conditions limites et certaines ne fleurissent pas ou produisent très peu de graines, sauf lors des étés austraux anormalement chauds. Une seule exception, Stellaria alsine, petite plante des milieux humides apparue à Crozet au début des années 1990, dont les modes de diffusion efficaces (graines facilement disséminées par le vent et multiplication végétative par fragmentation des rameaux) font peser une menace potentielle sur les tourbières et autres communautés des habitats humides de l'île de la Possession sur Crozet. Une telle menace pourrait se généraliser si, dans le cadre du réchauffement climatique contemporain, une élévation de température accroissait le pouvoir de dissémination des espèces végétales introduites.

L'influence des animaux introduits est d'ores et déjà beaucoup plus sensible, qu'il s'agisse d'insectes ou de mammifères. Les capacités adaptatives de la mouche bleue (Calliphora vicina), introduite à Kerguelen dans les années 1970, sont en effet étonnantes puisqu'après avoir passé les premières années dans les bâtiments chauffés de la base de Port-auxFrançais, cet insecte est rapidement parvenu à se reproduire à l'extérieur. Il concurrence aujourd'hui avec succès, lors des étés chauds, la mouche autochtone (Anatalanta aptera) que son aptérisme rend vulnérable face à la concurrence d'espèces invasives pourvues d'ailes. Quant au papillon des crucifères (Plutella xylostella), introduit avec les légumes frais destinés aux bases subantarctiques, il s'est largement répandu dans les années chaudes 1980-1990 et ses chenilles constituent aujourd'hui une menace pour le très emblématique chou des Kerguelen (Pringlea antiscorbutica).

Ce sont toutefois les mammifères introduits qui exercent l'influence la plus visible, sinon la plus profonde, sur le fonctionnement des écosystèmes subantarctiques. Le rat noir, qui se nourrit de jeunes oiseaux, a fait localement chuter la biodiversité aviaire à Crozet, mais ce sont les chats et surtout les lapins qui provoquent les plus graves dégâts. Dans les années 1950, quelques chats avaient été volontairement introduits à Kerguelen pour chasser les rats et les souris arrivés sur l'archipel en même temps que les baleiniers du XIX e siècle. En l'absence de prédateurs efficaces, ils se sont rapidement développés, jusqu'à atteindre une population de plusieurs milliers - il y aurait aujourd'hui environ 5000 chats à Kerguelen - et ces félins seraient responsables, sur le demi-siècle écoulé, de l'élimination de plusieurs millions de pétrels (oiseaux à reproduction lente dont les femelles ne pondent au mieux qu'un œuf par an).

La palme de la dégradation du milieu revient sans doute au lapin, introduit en 1874 par des marins pour constituer une réserve alimentaire en cas de naufrage. Depuis lors, l'espèce a pullulé sur Kerguelen et est à l'origine d'une dégradation généralisée au milieu : mise en coupe réglée des communautés végétales autochtones à chou des Kerguelen, azorelle et Acaena (fig. 6), destruction des sites de nidification des pétrels, érosion des sols. Dès les années 1950, l'introduction du virus de la myxomatose avait permis de réduire substantiellement l'effectif de lapins, mais ceux-ci sont progressivement devenus résistants au virus et il a fallu trouver une stratégie de rechange. À la fin des années 1980, l'introduction à Kerguelen de la puce du lapin, vecteur de la myxomatose, a contribué à une meilleure transmission du virus qui a fait chuter la densité de lapins sur l'archipel. Celle-ci demeure toutefois trop élevée pour permettre une restauration écologique des secteurs dégradés, et un programme d'éradication du lapin par empoisonnement a été 
lancé en 1992 sur trois îles. Mais la recolonisation végétale est lente, et des perturbations climatiques (sécheresses estivales) ont entraîné la mort des jeunes pieds de chou et d' Acaena, victorieusement concurrencés par le pissenlit.

Figure 6 : Érosion des sols et buttes relictuelles d'Acaena sur le littoral de Kerguelen Soil erosion and residual Acaena tufts near the Kerguelen coastline

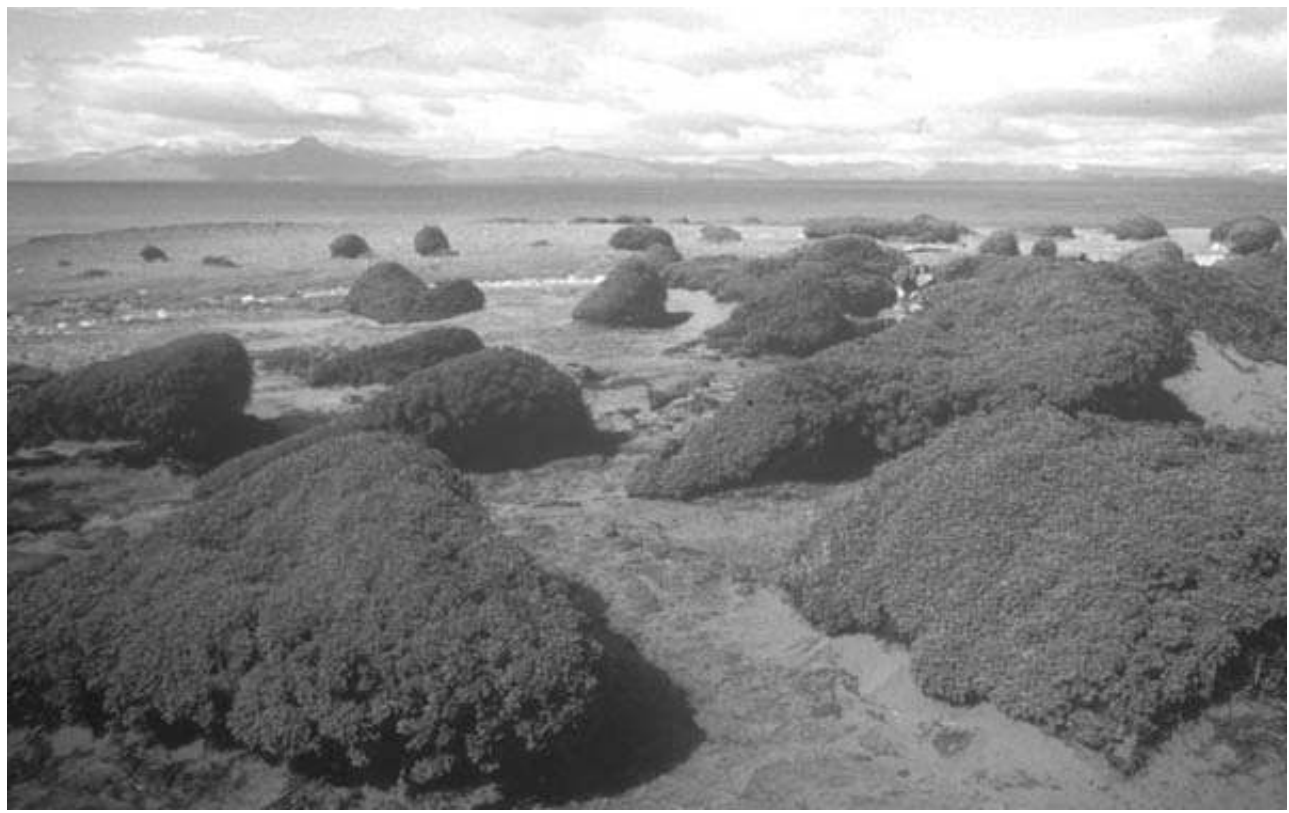

(๔ B. Labidoire)

27 Le retour de certaines espèces animales semble plus rapide que la restauration du couvert végétal. À Saint-Paul, les pétrels, qui avaient été pratiquement éliminés par le rat noir, sont rapidement revenus suite à l'éradication des rats par la dispersion d'appâts empoisonnés par hélicoptère. Sur l'île Amsterdam, où les vaches abandonnées par un éleveur à la fin du XIX ${ }^{e}$ siècle s'étaient multipliées, la mise en défens d'une moitié de l'île a permis le retour des populations d'albatros qui avaient fui, dérangées dans leur nidification par les vaches.

28 Si la restauration des communautés originelles semble possible, elle s'avère globalement lente et est aisément perturbée par des accidents climatiques. Un réchauffement pourrait favoriser les espèces tempérées introduites depuis deux siècles aux dépens de communautés subantarctiques uniques au monde. Un suivi de ces écosystèmes originaux s'impose donc, dans le prolongement des cinquante années d'observations dont disposent déjà les chercheurs français. Une telle banque de données est sans équivalent sous ces latitudes et justifie à elle seule le lancement, en 2001, d'un programme d'observation à long terme. Pilotée par Pierre Jouventin ${ }^{22}$, la zone-atelier subantarctique du CNRS (PEVSINSU) regroupe aujourd'hui les équipes françaises travaillant dans les terres Australes et Antarctiques Françaises où les scientifiques appellent à la création d'un parc naturel national.

Quittons les îles subantarctiques et notons que la connaissance de la biodiversité des milieux extrêmes s'enrichit régulièrement de nouvelles découvertes : dans le cadre de la commission GLACKIPR, une équipe slovène a ainsi étudié la microflore fongique des glaciers de la baie du Roi au Spitsberg ${ }^{23}$. Une grande quantité de microchampignons du genre Penicillium ont été découverts et identifiés, ce qui n'est pas surprenant puisqu'ils 
comptent parmi les plus présents dans l'atmosphère du globe. On regrettera que les auteurs aient laissé de côté les levures et les champignons noirs puisque, d'une part, ces populations microorganiques sont largement dominantes dans la glace (70 à $100 \%$ des souches isolées par ces auteurs!), et d'autre part, ce sont de meilleurs indicateurs des stratégies d'adaptation qu'elles ont dû développer pour s'adapter aux conditions de vie difficiles offertes par la glace.

\section{Les réseaux de recherche}

\section{Arctic Costal Dynamic (ACD)} d'informations sur les dynamiques littorales de la zone circum-arctique. Créé en avril 2000 avec le support de l'International Arctic Sciences Committee (IASC), ce réseau avait défini quatre grands objectifs sur les thèmes de la cartographie et classification des côtes arctiques; des processus littoraux des hautes latitudes; de la cartographie et de la quantification de la glace de sol; et de la définition de protocoles expérimentaux standardisés.

31 Ce réseau a permis la tenue de quatre conférences au cours desquelles de nombreuses communications ont porté sur l'érosion côtière, les budgets sédimentaires littoraux, la mise en commun des données permettant de mieux connaître le forçage environnemental commandant la dynamique littorale (vitesse du vent, niveau marin, fetch, etc.), l'optimisation des typologies des littoraux arctiques en vue de leur intégration dans des bases de données numériques, l'établissement d'une photothèque de géomorphologie littorale arctique consultable en ligne ou encore le développement d'un réseau de sites permettant le suivi sur le long terme des côtes. d'ores et déjà été publiés, ce qui montre la fécondité de ces regroupements internationaux, tant en terme d'échanges de données que de montage de programme de recherche communs.

(Contact : Dr Volker Rachold, Alfred Wegener Institute, [vrachold@awi-potsdam.de] ; [ http://www.awi-potsdam.de/www-pot/geo/acd.html].)

\section{ESF-SEDIFLUX}

Un nouveau réseau d'échanges de la Fondation Européenne pour la Science (ESF European Science Foundation) a été créé en janvier 2004 pour une durée de trois ans. Intitulé SEDIFLUX " Sedimentary source-to-sink fluxes in cold environments », ce réseau réunit une centaine de chercheurs européens, issus d'une vingtaine de pays, dont les préoccupations scientifiques ont lien avec les mutations des systèmes morphogéniques qui accompagnent les changements climatiques à la surface de la Terre. Les changements les plus spectaculaires devraient se produire dans les milieux soumis à des climats froids et l'Europe offre un large échantillon de sites où les relations climat/processus peuvent être étudiées et modélisées. Le réseau privilégie les approches pluridisciplinaires, en particulier les collaborations entre spécialistes des géosciences, écologues et biologistes. 
Le réseau est piloté par Achim Beylich (Université de Trondheim) et un comité de coordination à 10 membres. Il comprend différents groupes de travail et son activité principale consistera en la tenue de quatre ateliers (Saudarkrokur, Islande en juin 2004 ; Clermont-Ferrand en janvier 2005 ; Londres en décembre 2005 ; Trondheim en 2006), la publication d'articles, d'un manuel et la diffusion des activités du réseau sur différents supports multimédia (cédérom, lettres d'informations numériques, etc.).

(Contact: Achim Beylich [Achim.Beylich@ngu.no]; [http://www.esf.org/sediflux/ou], pour la France, Samuel Étienne [setienne@seteun.net].)

\section{International Tundra Experiment (ITEX)}

Dans le même esprit de collaboration scientifique pluridisciplinaire, l'International Tundra Experiment (ITEX) réunit depuis décembre 1990 des chercheurs issus de onze pays, dont l'ensemble des nations arctiques. Contrairement aux deux réseaux précédents, essentiellement basés sur l'échange de données, ITEX est un réseau de coopération autour de projets de recherche communs.

L'objectif de ses membres est d'examiner la réponse des espèces végétales arctico-alpines aux changements environnementaux, et plus spécifiquement les effets de l'accroissement des températures estivales. Cinquante sites répartis dans toute la zone arctique font aujourd'hui l'objet d'expérimentations.

Le modèle adopté par le protocole de recherche combine expérimentations à court et long terme et mesures stationnelles. Afin de minimiser au maximum les éventuelles interférences liées à l'appareillage technique de mesure et d'enregistrement des paramètres environnementaux, un protocole très simple a été retenu : celui-ci consiste en l'utilisation de cloches transparentes ouvertes au sommet qui provoquent un effet de serre au-dessus des plantes étudiées (fig. 7). Si les études sont avant tout menées au niveau de l'espèce (ceci permettant d'avoir recours à la génétique), des sauts d'échelle sont intégrés dans le traitement des données afin de pouvoir comprendre l'impact du forçage climatique au niveau de l'écosystème.

[http://www.itex-science.net] 
Figure 7 : Cloche transparente à sommet ouvert utilisée dans le cadre du programme ITEX, site d'Atagsuk, Alaska, $70^{\circ} \mathrm{N} 157^{\circ} \mathrm{W}$

Open-top clear chamber used in the ITEX program, Atagsuk site, Alaska, $70^{\circ} \mathrm{N} 157^{\circ} \mathrm{W}$

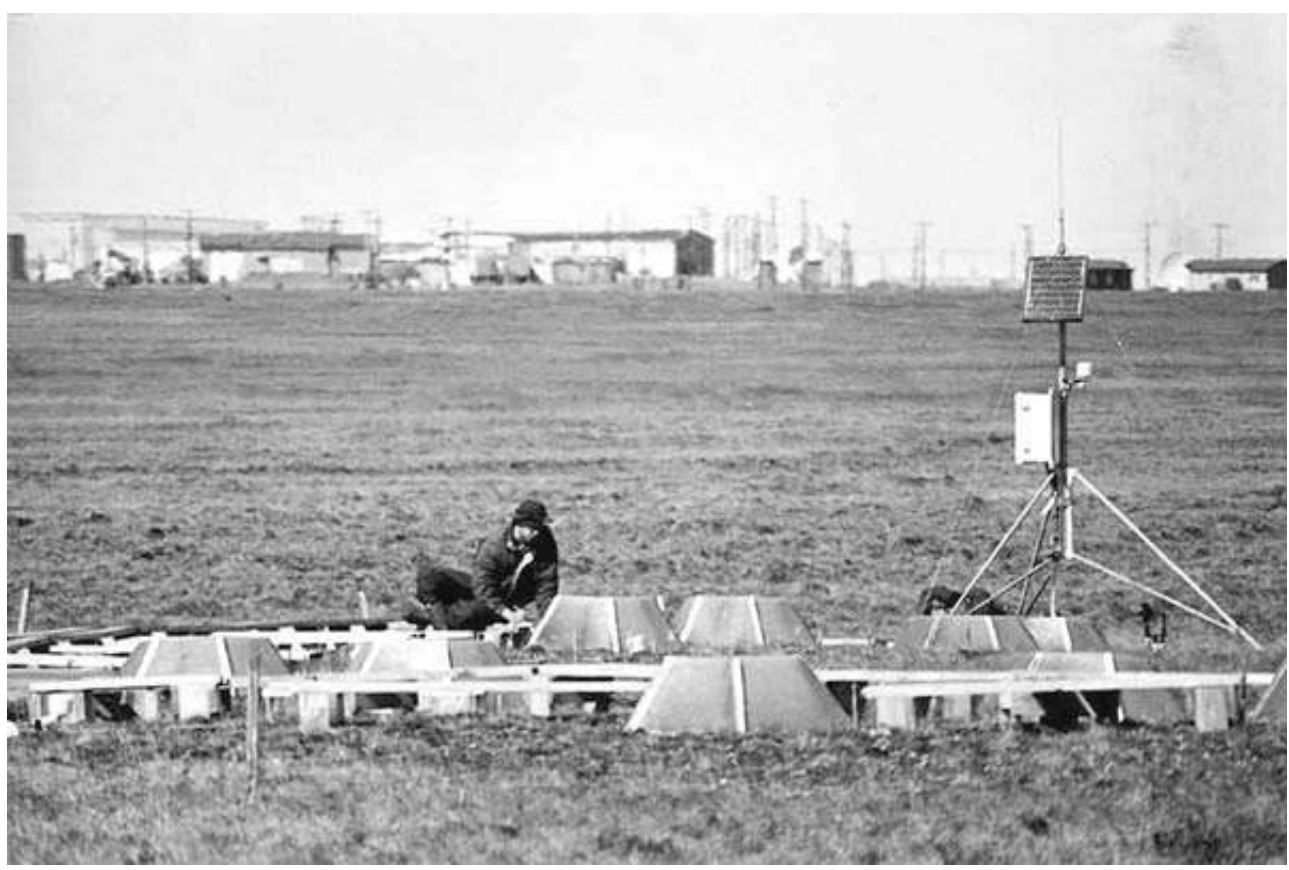

(c) R. Hollister)

\section{Les sites Web polaires}

41 La recherche scientifique progresse rapidement et les sites WEB apportent des outils précieux et indispensables pour recueillir des informations souvent dispersées à travers le monde. En voici une sélection utile pour le monde polaire.

- [http://npolar.no/nysmac/] Le site de NySMAC (The Ny-Ålesund Science Managers Committee) a été créé en 1994 pour facilité la coopération et la coordination des recherches à Ny-Ålesund. Cette station permanente du Svalbard accueille les bases scientifiques des Norvégiens, Français, Anglais, Allemands, Coréens, Chinois, Japonais...

- [http://www.transpolair.com] Site très riche sur les aventuriers des milieux polaires qui ont fait l'histoire de l'Arctique et de l'Antarctique (Amundsen, Andrée, Charcot, Nansen, Nobile, Scott, Shackleton, Victor...), mais aussi sur l'actualité des aventuriers contemporains. Les philatélistes y trouveront des informations précieuses.

- [http://www.arcus.org] ArcticInfo est administré par l'Arctic Research Consortium of the United States (ARCUS). Tout le monde peut souscrire à ArcticInfo en envoyant un courriel à arcticinfosub@arcus.org. Vous recevrez automatiquement les informations concernant des colloques, des postes vaquants dans les universités ou les organismes de recherches en rapport avec l'Arctique, des parutions d'ouvrages ou d'actes de colloques, concernant les milieux polaires.

- [http://www.qsl.net/f5nod/antarctica.html]

Site à partir duquel vous pouvez avoir accès à des webcams (pôle sud, Davis, Mawson, Neumayer, Casey, Macquarie) de même qu'à des informations météorologiques. 
- [http://www.kingsbay.no]

Site de la compagnie en charge de la concession de la presqu'île de Brøgger au Spitsberg avec des informations pratiques (avion...), une webcam, des informations météorologiques....

- [http://www.unis.no]

Site de l'université de Longyearbyen au Svalbard avec toutes les informations utiles pour les étudiants, mais également une webcam, des informations météorologiques du jour et un accès direct aux données journalières antérieures (température, vent, humidité, pression athmosphérique...).

- [http://npiweb.npolar.no/] Site de l'institut polaire norvégien (Norsk polarinstitutt) avec les informations pratiques, les activités, les publications, les cartes...

- [http://www.ifremer.fr/ifrtp/]

Site de l'institut polaire français Paul-Émile Victor (IPEV, anciennement Institut Français pour la Recherche et la Technologie Polaire IFRTP) sur lequel on trouve l'actualité des programmes scientifiques soutenus par cette agence de moyens, des informations sur les régions polaires en général et les bases scientifiques françaises en particulier.

- [http://www.awi-potsdam.de/] Site de l'institut polaire allemand (Alfred Wegener Institute for Polar and Marine Research).

- [http://www.nipr.ac.jp/] Site de l'institut polaire japonais avec notamment leurs publications.

- [http://www.nature.com/nature/focus/Icecores/]

Pages web de la revue Nature fournissant la liste détaillée des articles récents consacrés aux forages glaciaires dans l'Antarctique et au Groenland.

\section{L'actualité polaire}

\section{Premiers enseignements des forages EPICA et NorthGRIP}

Les glaces polaires constituent de remarquables archives climatiques et jusqu'à présent, les forages antarctiques profonds de Vostok ${ }^{24,25}$ ont fait autorité en la matière, avec 420000 ans d'histoire du climat. Ce record vient d'être pulvérisé au Dôme $\mathrm{C}$, dans $l^{\prime}$ Antarctique oriental ( $75^{\circ} 06^{\prime} \mathrm{S}, 123^{\circ} 21^{\prime} \mathrm{E}, 3233 \mathrm{~m}$ d'altitude), avec le deuxième forage EPICA (European Project for Ice Coring in Antarctica). Placé sous l'égide de la Fondation Européenne pour la Science et fortement soutenu par la Commission Européenne, ce projet fédère des laboratoires et opérateurs logistiques émanant de dix pays européens dont la France (avec le LGGE de Grenoble et le LSCE de Gif-sur-Yvette, et le soutien logistique de l'IPEV de Brest), le Royaume-Uni (British Antarctic Survey), l'Allemagne (Alfred Wegener Institut), ainsi que la Russie, la Norvège, le Danemark, l'Italie, la Suisse et le Benelux.

En juin 2004, la revue Nature a titré sur l'événement sous l'intitulé EPICA Adventure - a 740,000 - year record of Antarctic Climate $e^{26,27,28}$. Choisi en raison de l'épaisseur de la glace $(3300 \mathrm{~m})$, le dôme $\mathrm{C}$ - où est implantée la station franco-italienne Concordia - a largement tenu ses promesses, non sans quelques rebondissements. C'est en 1996 qu'a débuté le premier forage pour s'arrêter net deux ans plus tard en raison du blocage du carottier à -788 m. Un deuxième forage a été engagé en 2000-2001 et la profondeur de $3190 \mathrm{~m}$ a été finalement atteinte. Il reste encore $120 \mathrm{~m}$ à forer, et l'opération s'annonce difficile car cette glace basale est proche du point de fusion. Le forage EPICA devrait 
couvrir au total les derniers 800000 voire 900000 ans. À ce jour, 8 cycles climatiques, soit 740000 ans, sont déjà couverts par les premiers 3140 m (fig. 8).

Figure 8 : Cycles glaciaires couvrant les derniers 800000 ans (à gauche : enseignements des forages marins profonds - à droite : résultats du dernier forage glaciaire EPICA). À noter : le stade isotopique 11 (MIS 11), qui correspond à un interglaciaire présentant certaines similarités avec l'interglaciaire actuel

Glacial cycles of the past 800,000 years based on deep-sea cores (left) and EPICA ice core (right), with focus on Marine Isotope Stage 11 that bears similarities to the Present interglacial period

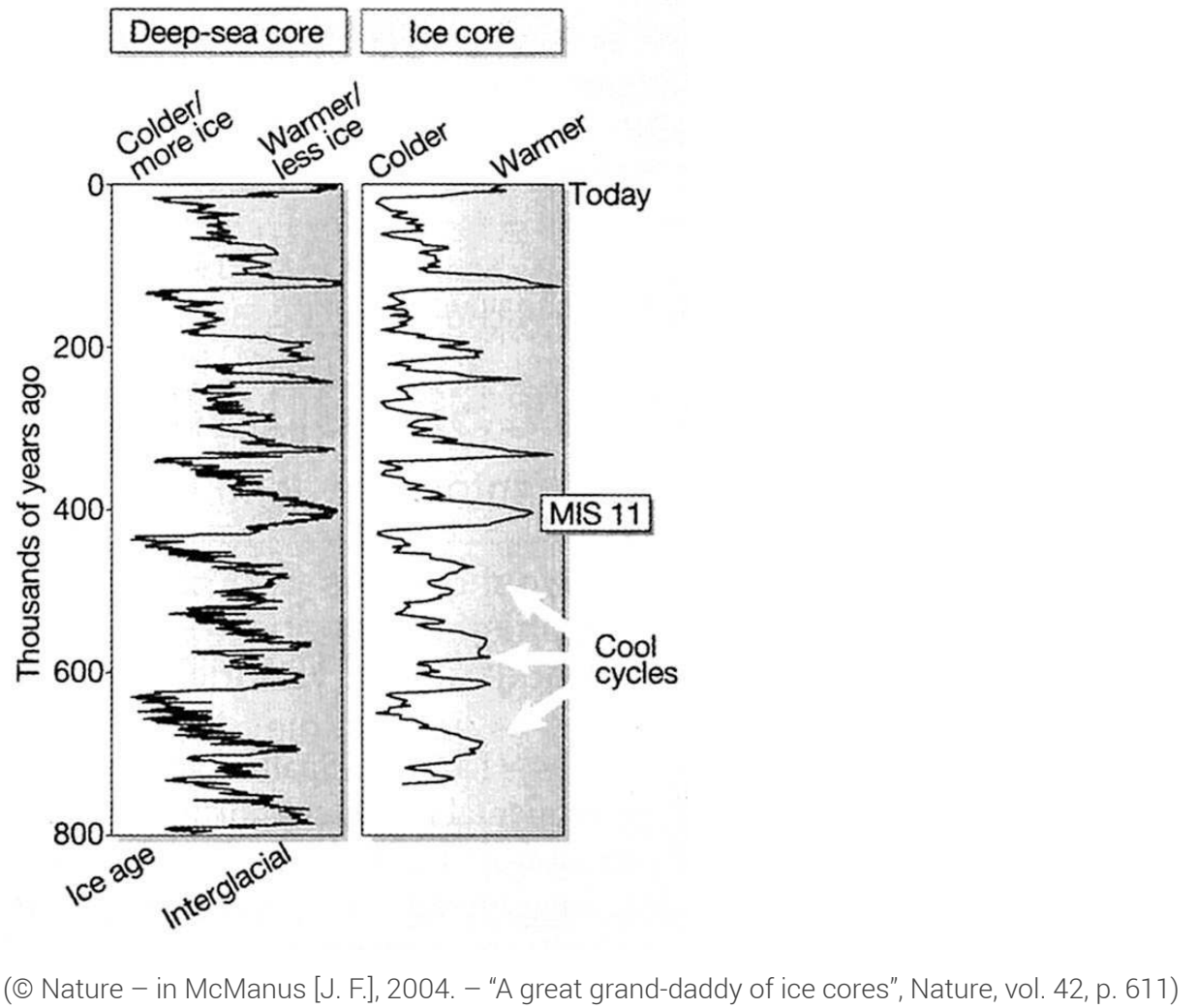

L'apport du forage EPICA à la connaissance de l'histoire du climat est double. Il révèle tout d'abord que les cycles climatiques n'ont eu ni la même amplitude ni la même périodicité avant et après 450000 ans. Alors que les 4 derniers cycles climatiques se caractérisent par une périodicité de 100000 ans et l'existence de puissants contrastes thermiques entre périodes glaciaires et interglaciaires, les cycles antérieurs ont vu s'installer une glaciation tous les 40000 ans; les cycles glaciaires avaient alors une moindre amplitude, de même que les interglaciaires, et ces derniers occupaient dans le cycle climatique un laps de temps beaucoup plus important que dans la période récente au cours de laquelle des périodes glaciaires de 100000 ans alternaient avec des interglaciaires courts, de 10000 à 15000 ans. Ce changement de rythme intervenu il y a quelque 400 millénaires demeure très largement inexpliqué.

Autre apport, un éclairage très précieux sur l'interglaciaire-clé du stade isotopique 11 , situé autour de -420000 ans, qui présente un intérêt tout particulier dans le contexte actuel du réchauffement climatique et des modèles prédictifs associés. Compte tenu de la parenté des configurations astronomiques, cet interglaciaire pourrait constituer le meilleur analogue de l'interglaciaire actuel ${ }^{29}$. Les bulles de glace renfermées par la glace montrent d'ailleurs des teneurs de l'atmosphère en $\mathrm{CO}_{2}$ et $\mathrm{CH}_{4}$ similaires à celles $\mathrm{du}$ 
niveau pré-industriel de notre interglaciaire. Mais alors que les interglaciaires qui ont précédé le nôtre ont duré entre 10000 et 15000 ans, l'interglaciaire du stade 11 a été exceptionnellement long puisqu'il a duré environ 28000 ans. L'Holocène ayant débuté il y a 10 000-12 000 ans, notre interglaciaire est donc, en théorie, susceptible de se prolonger pendant plus de 15000 ans. C'est compter sans l'effet de serre additionnel, marqué par la croissance exponentielle de la teneur de l'atmosphère en $\mathrm{CO}_{2}{ }^{30}$, qui risque de changer la donne. Si elle devenait suffisamment massive, la fusion des glaciers continentaux pourrait en effet réduire la salinité et donc la densité des eaux arctiques, empêchant la formation d'eaux profondes amorçant la pompe océanique mondiale qui nous restitue de la chaleur via le Gulf Stream. Et si les échanges énergétiques entre les Tropiques et les Pôles perdent de leur intensité, c'est bel et bien à un retour du froid anticipé qu'il faut nous attendre...

Trois mois après les premiers résultats du dernier forage antarctique EPICA, les scientifiques nous livrent les premiers enseignements du forage multinational NorthGRIP (North Greenland Ice Core Project) faisant suite aux forages GRIP et GISP2 évoqués dans une précédente chronique arctique ${ }^{31}$. Les déformations de la glace basale y brouillaient le message paléoclimatique concernant le dernier interglaciaire, ce qui avait motivé la mise en œuvre du forage NorthGRIP dans un secteur moins perturbé situé à $75^{\circ} 10^{\prime} \mathrm{N}$. L'épaisseur de l'inlandsis groenlandais atteint ici $3085 \mathrm{~m}$. Ce forage, qui a mobilisé chercheurs et techniciens américains, japonais et européens (avec une forte participation française), a débuté en 1996. Le socle rocheux a été atteint en juillet 2003 et les premiers résultats sont parus en septembre $2004^{32,33}$. Ce forage, qui offre une excellente résolution, couvre les derniers 123000 ans. Il intègre donc l'Holocène, le dernier glaciaire et une partie de l'interglaciaire éémien. Ce dernier apparaît légèrement plus chaud que l'actuel et est donc susceptible d'être utilisé comme analogue pour les scénarios prédictifs de réchauffement climatique par effet de serre additionnel. On retiendra notamment que le forage NorthGRIP met en évidence, non pas un passage brutal vers le dernier glaciaire, mais au contraire une décroissance très progressive des températures, qui s'est étalée sur 7000 ans (entre 122000 et 115000 BP).

\section{Préhistoire de l'Arctique russe}

Si l'on sait de longue date que les glaciations quaternaires ont très largement épargné les basses terres de l'Arctique russe, on a longtemps pensé que les rigueurs climatiques de la dernière période froide avaient empêché les hommes de s'y installer avant 13 000-14 000 BP. Il n'en est rien puisqu'une équipe russo-scandinave ${ }^{34}$ a récemment découvert par $66^{\circ}$ $34^{\prime} \mathrm{N}$ le gisement paléolithique de Mamontovaya Kurya, vieux de près de 40000 ans. Situé près de la pointe nord de l'Oural, sur les rives de la rivière Usa, ce site recèle de l'outillage lithique et un assemblage faunique dominé par le mammouth (en association avec le loup, le renne et le cheval). Une série de datations radiocarbone situées dans la fourchette 34 400-37 400 BP ont été obtenues sur le matériel osseux et une défense de mammouth gravée a livré un âge de $36660 \mathrm{BP}$. Les données polliniques restituent l'image d'un environnement steppique herbeux totalement dépourvu d'arbres. Gowlett ${ }^{35}$ souligne le fait qu'une telle adaptation au froid requiert de la part des communautés concernées un haut niveau d'organisation socio-économique et technologique. La période concernée marque la transition du Paléolithique moyen au Paléolithique supérieur, et en l'absence de restes humains, la question se pose de savoir si les occupants du site de Mamontovaya Kurya étaient des Néanderthaliens (Homo sapiens neanderthalensis) ou des hommes 
anatomiquement modernes (Homo sapiens sapiens). Compte tenu de la robustesse et des capacités adaptatives des Néanderthaliens, la première hypothèse est parfaitement plausible. S'il s'agit au contraire d'hommes modernes, cela implique de leur part une surprenante rapidité de déplacement en direction du nord puisqu'ils venaient tout juste de mettre le pied dans le Sud-Est de l'Europe.

\section{Ouvrages récents}

\section{Des livres sur les conquérants des pôles}

Parmi les ouvrages récents, postérieurs à la dernière chronique arctique (Norois, $\mathrm{n}^{\circ} 186$, 2000), notons la sortie du dictionnaire de Michel d'Arcangues ${ }^{36}$. Il permet de remettre à sa juste place tous les noms de l'épopée polaire. Nous y trouvons des explications toponymiques difficilement accessibles, des renseignements inédits sur l'histoire des grands noms mais également des éléments précieux sur les anonymes de l'exploration polaire, bref un indispensable outil de travail. Car ici revient tout le mérite de Michel d'Arcangues qui a réussi à sortir des oubliettes de l'histoire des personnalités fortes et des récits passionnants. 191 personnages ont retenu l'attention de l'auteur. De Louis Amédée Ferdinand François de Savoie, duc des Abruzzes, dont l'expédition à la conquête du pôle nord est passée tout près de l'exploit en 1900, à Dimiti Mikhaïlov Zyryan, personnage du xviie siècle qui consacra sa vie à l'exploration de la Sibérie orientale, tous ont leur notice biographique. S'il est heureux que des héros comme E. H. Shackelton, R. G. Amundsen, J.B. Charcot, J. Franklin, F. Nansen, R. F. Scott... bénéficient de plusieurs pages, d'autres grands noms sont moins favorisés (P.-É. Victor...). En revanche, on regrette vivement des oublis malheureux (J.-F. de Galaup de La Pérouse, Charles Rabot, les frères Rallier du Baty, L. Delisle de la Croyère...). Par ailleurs, comment justifier les choix de retenir Helen Thayer et Liv Arnesen, et passer sous silence l'extraordinaire expédition de Madeleine Griselin? Pourquoi consacrer quatre pages pour Thomas Edge, baleinier anglais, et rien écrire sur son homologue basque et contemporain Jean Vrolicq? Pourquoi ne pas parler des alpinistes français au Spitsberg: Martin, Pommier et Valette en 1946 et surtout Maillard, Cabanes, Daguillon, Dameron, Gendron et Passini, en 1950, qui baptisèrent le sommet de l'archipel du Svalbard « mont Général Perrier ", ancien président de la Société de géographie de Paris, qui venait de décéder ? On le voit, Michel d'Arcangues ne favorise ni la France ni les explorateurs français, et la liste des oubliés n'est pas exhaustive. Cela dit, la moisson n'était pas une entreprise facile et cet ouvrage permet de combler une lacune dans la bibliothèque polaire. L'importante bibliographie $(37$ p.) permettra à tous ceux qui veulent en savoir plus d'aller étancher leur soif de connaissance. Les éternels insatisfaits attendront la prochaine édition revue, augmentée et améliorée de belles cartes.

Dans le même registre, Kåre Berg vient de publier un livre ${ }^{37}$ superbement illustré sur les héros du panthéon polaire norvégien. L'auteur fut directeur du célèbre musée Fram de Bygdøy. La couverture utilise la photographie de Roald Amundsen au cours de son expédition vers le pôle Nord en 1925, mais c'est par la traversée à ski du Groenland d'est en ouest sur $560 \mathrm{~km}$, dirigée par Fridtjof Nansen en 1888, que le livre commence. Le deuxième chapitre relate l'aventure du Fram dans les eaux arctiques entre 1893 et 1896 et la tentative de Fridtjof Nansen et de Fredrik Hjalmar Johansen de rejoindre le pôle Nord à ski. Ils arrivèrent à la latitude jamais atteinte jusqu'alors de $86^{\circ} 14^{\prime} \mathrm{N}$ le 7 avril 1895, à 
seulement $450 \mathrm{~km}$ du pôle. Le chapitre se poursuit sur leur histoire singulière durant 462 jours pour rejoindre la terre ferme, leurs rencontres avec les ours polaires, leur hivernage, et enfin leur retour à Vardø le 13 août 1896, le même jour où le Fram était libéré de la banquise au large du Svalbard, après 1056 jours prisonnier des glaces. «Their timing was perfect. » écrit l'auteur. Le troisième chapitre est consacré à l'exploration et à la cartographie des territoires du grand nord canadien et de la deuxième expédition du Fram . C'est l'occasion de rendre hommage à Otto Sverdrup, compagnon de Nansen lors de la traversée du Groenland et, surtout, devenu commandant du Fram lors de l'expédition de Nansen et de Johansen vers le pôle. L'expédition de Sverdrup, sur le Fram transformé, entre 1898 et 1902, permit la cartographie de $200000 \mathrm{~km}^{2}$ et de collecter des échantillons qui furent ensuite analysés jusqu'en 1930. Le quatrième chapitre raconte les aventures antarctiques de Carl Anton Larsen entre 1892 et 1903 qui identifia des affleurements de roches sédimentations sur l'île de Seymour et les conséquences paléogéographiques que cette découverte impliquait ; mais également l'aventure du Belgica, bateau commandé par Adrien de Gerlache de Gomery ${ }^{38}$, lors de son expédition entre 1897 et 1899, à laquelle participa comme subalterne Roald Amundsen (fig. 9). La transition est toute trouvée pour introduire le grand héros norvégien. Entre 1897, comme membre de l'expédition antarctique, et sa mort tragique le 18 juin 1928 en portant secours à Umberto Nobile, Roald Amundsen a découvert le passage du Nord-Ouest à bord du Gjøa, a atteint le premier le pôle Sud, a commandé l'expédition à bord du Maud à travers le passage du Nord-Est, a survolé le pôle Nord. Tous ces exploits pour un seul homme méritaient bien évidemment plusieurs chapitres. Le cinquième est donc logiquement consacré au passage du Nord-Ouest en 1906, le sixième à la conquête du pôle Sud le 15 décembre 1911, un mois avant l'arrivée de l'expédition anglaise menée par Scott le 17 janvier 1912, dont la fin tragique est également relatée. Le septième chapitre décrit le passage du Nord-Est avec le Maud, baptisé ainsi en l'honneur de la reine de Norvège. La traversée se déroula entre 1918 et 1920. À bord, un autre grand nom du panthéon polaire norvégien fait son apparition, Harald Ulrik Sverdrup, scientifique reconnu et dont les travaux en météorologie et océanographie firent dates. Il devint directeur de l'institut polaire norvégien. Cette institution lui rend toujours hommage en conservant son effigie, aux côtés de celles de Nansen et d'Amundsen, sur ses publications. Le huitième chapitre est consacré à la navigation du Maud dans les eaux arctiques entre 1922 et 1925 et aux premiers contacts d'Amundsen avec l'aviation. Le neuvième chapitre raconte comment Amundsen s'attaqua au pôle Nord par les airs avec deux Dornier (N24 et N25) en 1925, sans succès, puis à bord du dirigeable Norge, toujours au départ de Ny-Ålesund au Spitsberg. Cette fois, l'expédition d'Amundsen, avec l'Américain Lincoln Ellsworth, qui apportait sa fortune, et l'Italien Umberto Nobile, qui venait avec son dirigeable, fut une réussite. En 1926, le 11 mai, le Norge s'envolait de la presqu'île de Brøgger avec seize hommes à bord, survola le pôle Nord dès le lendemain et acheva son périple sur les côtes de l'Alaska à 90 $\mathrm{km}$ de Nome le 14. Ce chapitre s'achève en relatant le funeste sauvetage de Nobile en 1928. Outre Amundsen, ce ne sont pas moins de 16 hommes qui périrent pour secourir Umberto Nobile et ses hommes. Ce livre magnifiquement illustré par des cartes et des photographies se termine par l'évocation de la construction du musée à la gloire du Fram. 
Figure 9 : Statue d'Amundsen à Ny-Ålesund, Spitsberg Amundsen's statue in Ny-Ålesund, Spitsbergen

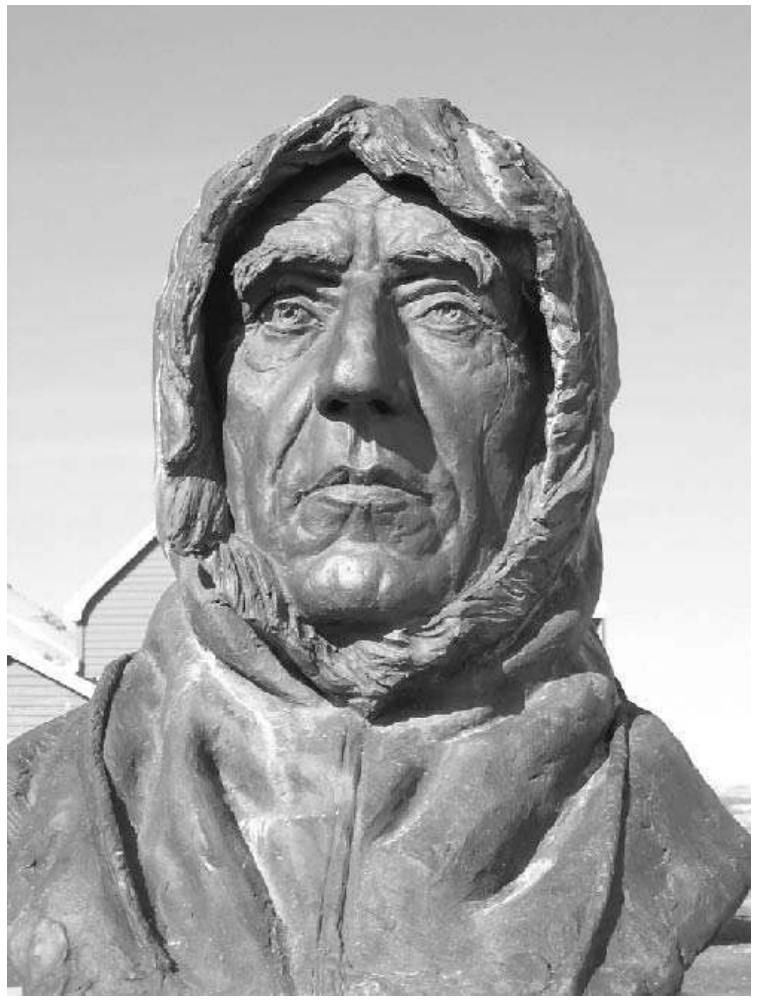

(C) D. Mercier)

l'expédition intitulée À la découverte de la Sibérie. Géographes et voyageurs français de Pierre Le Grand à nos jours. Cette publication bilingue franco-russe de soixante-quatre pages permet de souligner la coopération des géographes français et russes au cours de l'exploration de la Sibérie. Le Fonds polaire Jean-Malaurie, hébergé au Muséum, a fourni la précieuse documentation. Cette superbe publication reprend les épopées des Français à partir de 1653 avec Pierre-Martin de la Martinière. Puis ce sont Philippe Avril, les frères Delisle, les encyclopédistes Voltaire, Buffon, Diderot, Jean Chappe d'Auteroche, Eugène Patrin, JeanFrançois Galaup de la Pérouse, Jean-Baptiste Barthélémy de Lesseps, Adelbert von Chamisso, Abel Dupetit-Thouars, Lise Cristiani, Alphonse Pinart, Jules Verne, Joseph Martin, Edmond Cotteau, Edgar Boulangier, Charles Rabot, Charles Vapereau, Joseph de Baye, Paul Labbé, Louis Marin, Albert Bordeaux, Louis Philippe Robert Duc d'Orléans, Charles Bénard, George Montandon, André Leroi-Gourhan, qui bénéficient d'une attention particulière. Jean Malaurie, commissaire scientifique de l'exposition, en profite pour rappeler la création de l'Académie polaire d'État de Saint-Pétersbourg dont il est le président d'honneur à vie.

51 Si Umberto Nobile est bien connu de l'histoire polaire italienne, son compatriote le Duc des Abruzzes l'est beaucoup moins. Les éditions Economica rééditent le récit ${ }^{39}$ de ce pionnier de la conquête du pôle Nord, dans une collection baptisée Polaires ${ }^{40}$ et dirigée par Jean Malaurie ${ }^{41}$. Cette expédition dépassa en avril 1900, le record de Nansen, en atteignant la latitude de $86^{\circ} 34^{\prime} \mathrm{N}$ (fig. 10) et apporta de nombreux éléments scientifiques 
sur ces contrées encore mal connues. Ce livre veut réhabiliter cette expédition et au-delà le rôle joué par l'Italie dans l'exploration polaire.

Figure 10 : Route suivie vers le pôle Nord par une partie de l'expédition du Duc des Abruzzes en traîneau en 1900

Route by sled to the North Pole from members of Duc des Abruzzes expedition in 1900

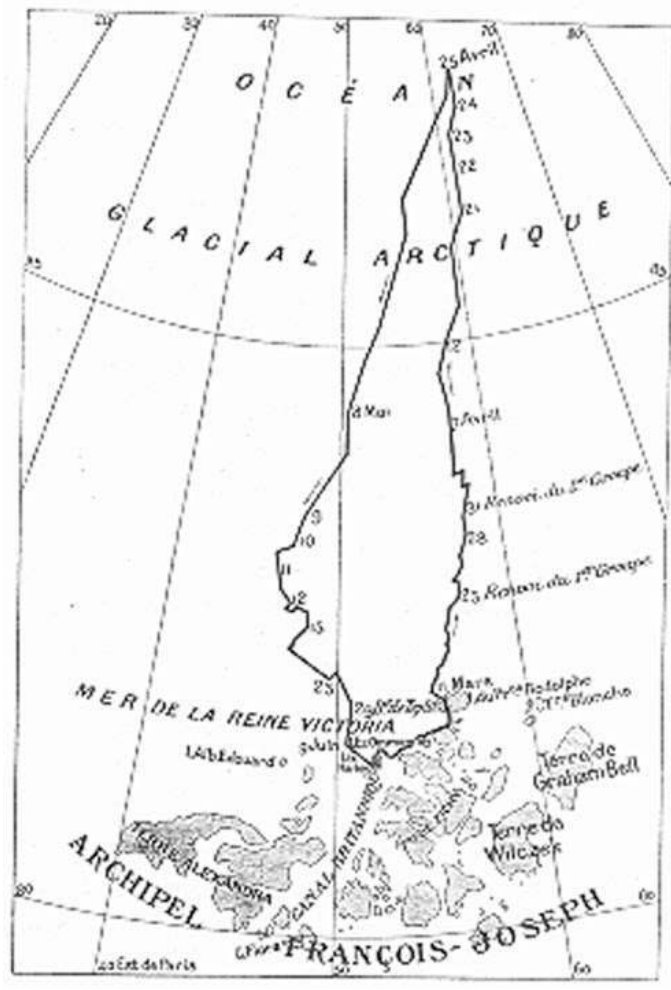

Un magnifique livre bilingue (norvégien-français) sur l'expédition de la corvette $L a$ Recherche a été publié en $2002^{42}$. L'expédition de La Recherche a été menée entre 1838 et 1840 vers le Spitsberg avec Paul Gaimard comme chef. Celui-ci avait servi comme chirurgien sous le commandement de Dumont d'Urville lors d'un tour du monde quelques années plus tôt. Cette expédition soutenue par la France, soucieuse à cette époque de devenir une grande nation sur le plan de la recherche scientifique, donna lieu en son temps à vingt-six volumes d'articles regroupés par thèmes (astronomie, hydrographie, météorologie, magnétisme terrestre, aurores boréales, géologie, géographie physique, géographie botanique, botanique et physiologie, histoire de la Scandinavie, littérature scandinave...) et cinq grands atlas d'illustrations. Les comptes rendus de voyage ont été rédigés par l'éminent homme de lettres et scandinaviste du XIX ${ }^{e}$ siècle, Xavier Marmier. Né à Pontarlier, il légua sa bibliothèque à cette ville, où l'on peut encore y consulter ses œuvres. Le présent ouvrage veut sortir des oubliettes de l'histoire cette fabuleuse aventure humaine en relatant l'expédition et en reproduisant de splendides lithographies originales d'Auguste Mayer (fig. 11). Le travail iconographique de ce Brestois se révèle d'une extrême richesse et offre un support documentaire unique sur les paysages et la vie quotidienne en Norvège au début $\mathrm{du} \mathrm{xIx}^{\mathrm{e}}$ siècle. On admire également les œuvres de François-Auguste Biard, qui rejoignit l'expédition en 1839 au Spitsberg, en compagnie de la jeune Léonie d'Aunet, qui elle même nous relata ses sentiments sur le grand nord dans un ouvrage publié pour la première fois en 1854 et souvent réédité ${ }^{43}$. Les fresques de Biard sur la baie de la Madeleine, au nord-ouest du Spitsberg, sont très réalistes et l'on 
peut aussi en admirer certaines, en grandeur nature sur les murs du Musée de Minéralogie du jardin des plantes de Paris. Cette grande expédition méconnue, associant chercheurs français et scandinaves, est une des premières expéditions scientifiques pluridisciplinaires et internationales à laquelle ce livre rend superbement hommage.

Figure 11 : La Recherche à Bellsund, Spitsbergen (lithographie d'Auguste Mayer) La Recherche in Bellsund, Spitsbergen (lithography by Auguste Mayer)

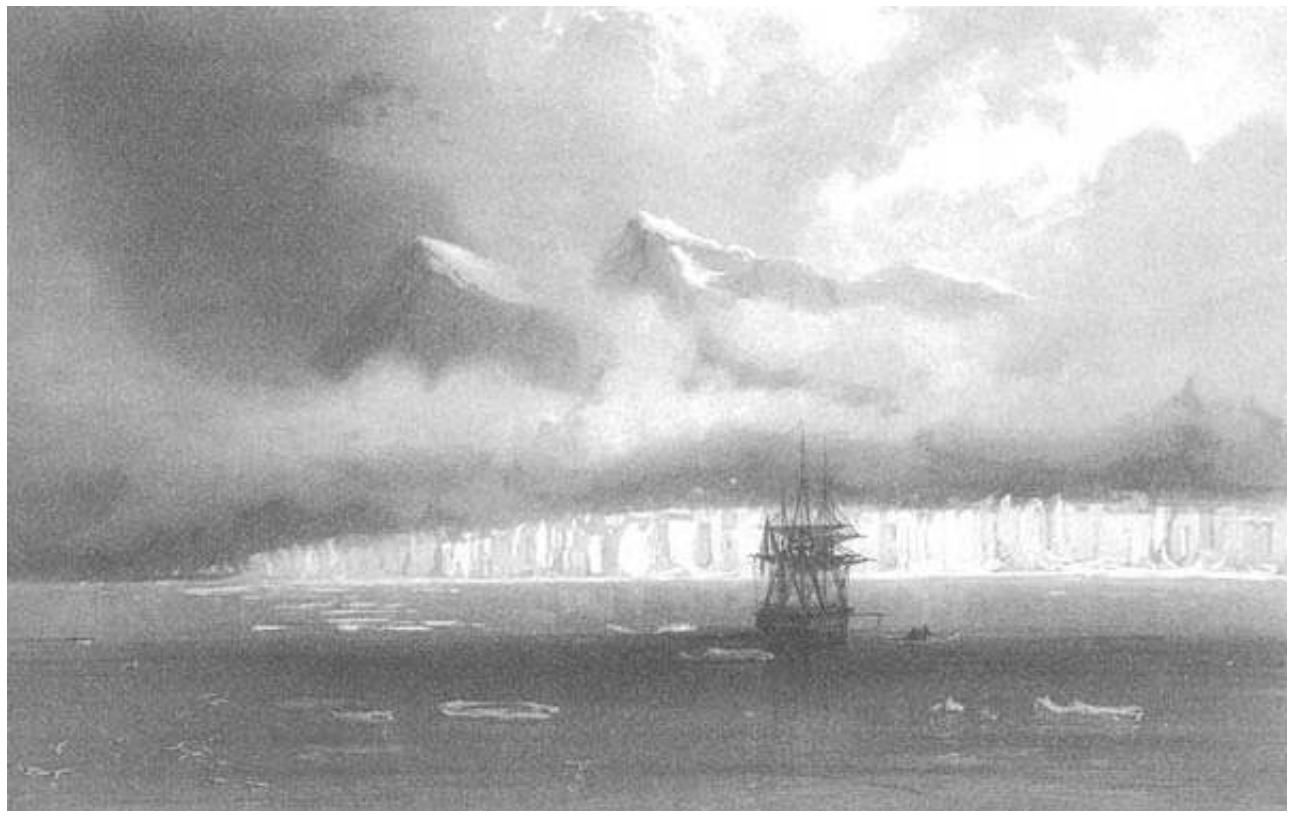

Les éditions du Septième continent associées au Seuil, publient un livre novateur intitulé Rêveurs de Pôles. Les régions polaires dans l'imaginaire occidental ${ }^{44}$. Emmanuel Hussenet, son auteur, bien connu des passionnés du Spitsberg, nous livre un ouvrage moins nombriliste que les précédents ${ }^{45}$. L'excellente iconographie bien sélectionnée facilite la lecture passionnante. Le corpus retenu par Emmanuel Hussenet est classique quand il aborde le Pôle antique avec Pythéas, Strabon, Virgile. Mais les pages les plus originales sont celles sur l'imaginaire polaire dans la littérature avec Jonathan Swift, Nicolas Edme Restif de La Bretonne, Mary Shelley, Edgar Allan Poe, Jules Verne, Victor Hugo... La peinture (où l'on retrouve entre autres François-Auguste Biard), le cinéma (Nanouk l'esquimau, Agaguk, Atanarjuat...), la littérature enfantine (Apoutsiak, Caroline au Pôle Nord...), offrent autant de support pour une analyse des pôles dans l'imaginaire occidental.

\section{De nombreux ouvrages récents sur les changements climatiques dans l'Arctique ${ }^{46}$}

L'ouvrage sur les bilans de masse de la cryosphère ${ }^{47}$ se concentre sur deux composantesclé de cet environnement sensible : la glace continentale (calottes polaires et glaciers) et la glace de mer (notamment les banquises). Celles-ci constituent des indicateurs des changements climatiques, aussi bien à court qu'à long terme. Rédigées par une équipe de 23 chercheurs, les approches sont autant méthodologiques que thématiques. Les inlandsis (Antarctique et Groenland) sont, bien entendu, à l'honneur, mais les autres glaciers ne sont pas oubliés. 
Julian Dowdeswell, de l'université de Cambridge, directeur du célèbre Scott Polar Research Institute, et Michael Hambrey du centre de glaciologie de l'université d'Aberystwyth (Pays de Galles), ont fédéré leurs longues expériences polaires pour nous livrer un magnifique ouvrage sur les îles de l'Arctique ${ }^{48}$. Le public visé n'est pas celui des spécialistes de ces contrées, qui selon leurs centres d'intérêts trouveront toujours quelques lacunes, mais bien au contraire le lecteur curieux non initié. Les illustrations en couleurs, essentiellement des photographies issues des fonds personnels des auteurs, accompagnent un texte clair. Les îles étudiées, outre le Groenland, appartiennent à l'archipel canadien, au Svalbard et à l'Arctique russe. On notera tout de même un déséquilibre dans l'iconographie au bénéfice du Spitsberg. Il ne s'agit pas d'une étude régionale, mais d'une approche des questions d'actualité par grands thèmes. Après un premier chapitre introductif sur la géographie des îles arctiques, dont les limites ne sont pas très clairement définies, le deuxième chapitre retrace l'évolution géologique de ces îles depuis 3,8 milliards d'années, âge des plus vieilles roches connues et retrouvées au Groenland. Le troisième chapitre décrit les ambiances climatiques actuelles et les changements climatiques survenus depuis 100000 ans. En écho, le chapitre suivant aborde la question des masses glaciaires et de leurs réponses aux changements climatiques. Le cinquième chapitre quitte les îles proprement dit et aborde les variations saisonnières des glaces de mer. Le sixième chapitre revient sur les phénomènes périglaciaires continentaux désormais bien connus. Les côtes, rivières et lacs font l'objet du septième chapitre, de même que la faune et la flore sont présentées dans le chapitre suivant. Ensuite, le neuvième chapitre examine la manière dont les hommes se sont adaptés à cet environnement hostile et aborde les questions écologiques posées par la fréquentation touristique. Pour conclure, le dernier chapitre présente rapidement les mesures de protection de l'environnement et les possibles conséquences des changements climatiques. En somme beaucoup de thèmes, trop diront certains, pour un seul ouvrage mais si agréable à lire.

\section{L'Antarctique vu de l'espace}

56 C'est un ouvrage à la fois accessible et magistral que Frédérique Rémy, directeur de recherche au CNRS, a récemment publié sur le continent antarctique ${ }^{49}$. Ce livre très informatif et superbement illustré fait avec beaucoup d'équilibre et de clarté le point de l'état des connaissances sur le continent blanc, véritable «mémoire de la terre ", à la fois " témoin et acteur du système climatique ». Comme le souligne l'auteur, l'image offerte actuellement par l'Antarctique n'est qu'un instantané, "un état transitoire à partir duquel il faudra restituer le passé et anticiper le futur ». Il existe pour cela une grande diversité d'approches, mais l'accent est mis dans ce livre sur les avancées liées à l'utilisation de la télédétection. La technique est toujours ici au service de problématiques scientifiques dont chacune appelle des options spécifiques (choix de capteurs spatiaux, de fréquences d'observation et de résolution spatiale). Les avancées sont nombreuses, concernant notamment la dynamique du climat et des glaces de mer, le drainage de la glace continentale (icestreams et ice-shelves) et la distribution des lacs et écoulements sousglaciaires.

57 Tout en mettant l'accent sur l'apport de la télédétection, F. Rémy brosse un véritable portrait du continent antarctique et des chercheurs qui y travaillent, depuis l'époque fondatrice de l'Année Géophysique Internationale (1957-1958) jusqu'à celle, toute récente, 
de la construction de la station Concordia et du forage EPICA au Dôme C (fig. 12). Sont abordés tour à tour : la «machine antarctique » actuelle (dans ses dimensions climatique et glaciologique) ; l'histoire du continent (du super-continent Gondwana à la formation de l'inlandsis, il y a plus de 15 millions d'années) ; les archives glaciaires (excellente synthèse à compléter par les données toutes fraîches des forages EPICA et NorthGRIP, cf. supra); le système austral (océan-atmosphère) ; la dynamique des calottes polaires (nécessaire prise en compte de la rhéologie de la glace dans l'interprétation des forages et la mise au point de modèles prédictifs); les interactions entre cryosphère et changements climatiques (compte tenu de l'inertie de l'inlandsis antarctiques, les décharges d'icebergs actuelles pourraient n'être qu'un effet retardé du réchauffement holocène, et non la conséquence immédiate du réchauffement contemporain).

Figure 12 : Forages et raids antarctiques effectués dans le cadre du projet EPICA

Ice cores and Antarctic raids realized by the partners of the EPICA Project

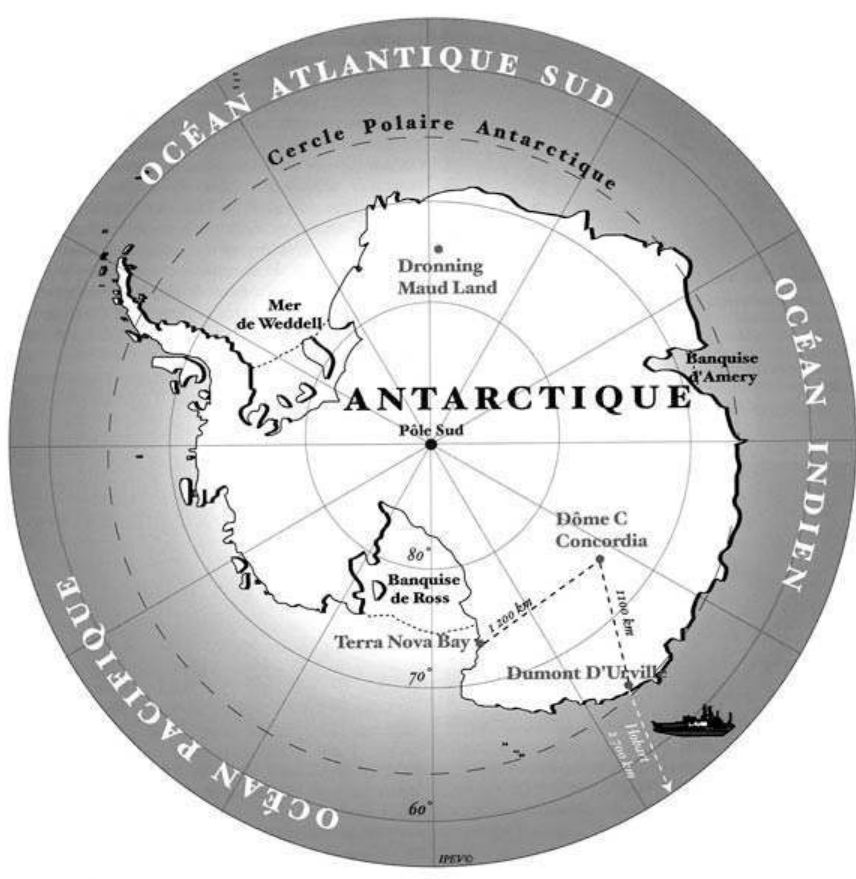

(๔) IPEV - Institut Polaire Paul-Émile-Victor, Brest - in Rémy (F.), 2003. - L'Antarctique - la mémoire de la Terre vue de l'espace, CNRS Éditions, p. 31).

Au total, si l'ouvrage de F. Rémy emporte l'adhésion, c'est non seulement en raison de ses qualités formelles, mais parce qu'il a un ton qui mêle avec bonheur enthousiasme et humilité : «L'ère du spatial a offert une vision tout à fait nouvelle de ce continent », qui a « mis en évidence une machine aux rouages complexes, loin d'être tous compris». « Nous ne savons toujours pas si le volume de l'Antarctique, actuellement, augmente ou diminue » (p. 23-24). Même si l'auteur a l'honnêteté rare d'admettre que «la fonte de la calotte polaire antarctique est loin d'être à l'ordre du jour ", les enjeux existent qui justifient pleinement un effort accru de recherche dans le cadre des grands projets internationaux d'observation du continent. L'achèvement prochain de la construction de CONCORDIA, base permanente franco-italienne implantée à Dôme C (fig. 12) et les 
résultats exceptionnels du dernier forage EPICA (cf. supra) témoignent de l'engagement des chercheurs français et européens dans cette exaltante aventure scientifique.

\section{Deux thèses de géographie sur l'Islande}

La recherche géographique française en Islande est une longue tradition, que les lecteurs de Norois connaissent bien. Succédant aux travaux pionniers des années cinquante (Bout et al., 1955) $)^{50}$, deux thèses ${ }^{51}$ ont été soutenues la même année sur des sujets complètement différents mais ayant comme points communs des thématiques de recherche d'une grande actualité et comme cadre spatial l'Islande. La thèse d'Armelle Decaulne nous entraine dans les fjords du nord-ouest de l'île et appréhende les relations entre dynamiques de versant (notamment les avalanches et les coulées de débris) et occupation humaine. Cette dualité aléa-vulnérabilité est, bien entendu, la base de la recherche cindynique en plein essor ces dernières années dans la communauté géographique. Cette belle thèse bien illustrée montre combien les dynamiques géomorphologiques sont éloignées des préoccupations des habitants de ces fjords, où les contraintes de site l'emportent sur la logique d'une implantation sans risque.

travail de Samuel Étienne, récemment publié dans une version largement remaniée ${ }^{52}$ s'inscrit dans un courant international de réflexion sur la hiérarchie des processus à l'œuvre dans les milieux froids. La thèse qu'il défend est celle de la place première des processus de biométéorisation à la surface des affleurements basaltiques dans l'évolution des milieux froids. Jusqu'à présent négligés dans l'approche géomorphologique zonale, les processus azonaux de ces milieux sont désormais en voie de réhabilitation ${ }^{53}$.

\section{Numéros spéciaux de revues}

61 Les éditions du CNRS et Economica se sont associées pour la publication du dernier volume de la revue internationale d'études arctiques, Inter-Nord ${ }^{54}$. Fidèle à sa tradition, cette revue publie des articles de sciences de la terre (ici sur les glaciers du Spitsberg et de l'arctique canadien); de sciences historiques (cinq articles dont un fait écho au livre sur l'expédition scientifique française de La Recherche entre 1838 et 1840, un autre sur Victor Hugo « esquimologue » qui lui aussi fait écho à cette même expédition dans la mesure où le célèbre écrivain a bien connu Léonie d'Aunet; de sciences de la vie (trois articles); de sciences sociales (neuf articles). Une rubrique est consacrée aux instituts de recherche arctique et une autre aux profils de personnalités arctiques. Un cahier spécial est dédié au Nunavik avec sept contributions. Ce gros volume avec illustrations en couleurs fait honneur à Jean Malaurie qui dirige cette publication depuis sa création en 1960, qui en est à sa vingtième livraison, soit 6000 pages et 250 auteurs.

La revue Chemins d'étoiles éditée par Transboréal a consacré son dixième numéro au Grand Nord $^{55}$. Les articles sont superbement illustrés. Les auteurs, aux noms plus ou moins illustres (Jean Malaurie, Jean-Louis Étienne, Joëlle Robert-Lamblin, Christian Mallet, Béatrice Collignon, Éric Brossier, Emmanuel Hussenet...), livrent des articles, dialogues, et informations très utiles. En écho à l'épopée de La Recherche, on notera l'article passionnant de Vérane Partensky "peindre la glace» dans lequel il est notamment question des œuvres de François-Auguste Biard sur le Spitsberg. 


\section{Autres livres polaires}

63 Le philosophe Michel Onfray emmène son père en terre de Baffin et nous rapporte une réflexion passionnante sur la temporalité publiée chez Grasset ${ }^{56}$.

Le pôle Sud et les expéditions scientifiques inspirent la romance à Marie Darrieussecq ${ }^{57}$. Avant elle, Jean Echenoz nous avait convié à une chasse au trésor au milieu de la banquise arctique. En guise de trésor polaire, l'auteur reçut le prix Goncourt $1999^{58}$.

Les expéditions polaires, souvent dramatiques, peuvent également inspirer un humour décalé sous la plume de Robert Benchley, chroniqueur de presse américain des années 1920, dont les écrits viennent d'être réédités ${ }^{59}$.

\section{NOTES}

1. Voir, par exemple, RAвот (C.), 1905. - « Mouvements géographiques », La Géographie, 12, p. 328-353.

2. Voir la recension des thèses françaises de géographie conduites en Arctique dans MERCIER (D.), 2003. - « Les géographes français et les milieux polaires et subpolaires ", Bulletin de l'Association de Géographes Français, 4, p. 407-416.

3. MAHER (P. T.), STEel (G.), MCinTosh (A.), 2003. - «Examining the experiences of tourists in Antarctica », International Journal of Tourism Research, 5, p. 59-62.

4. SERRANO CAÑADAS (E.), 2002. - « Hielo, montañas, mar y fauna : el turismo en las islas Shetland del Sur (Antartida maritima) », Revue de Géographie Alpine, 1, p. 9-23.

5. INGHAM (R. J.), SUMmERS (D.), 2002. - « Falkland Islands Cruise Ship Tourism : an overview of the 1999-2000 season and the way forward ", Aquatic Conservation: Marine and Freshwater Ecosystem, 12, p. $145-152$.

6. GREMMEen (N. J. M.), SMITH (V. R.), van TONGEREN (O. F. R.), 2003. - « Impact of trampling on the vegetation of subantarctic Marion Island ", Arctic, Antarctic, and Alpine Research, 35, p. 442-446.

7. KAAE (B. C.), 2002. - « Nature and Tourism in Greenland», dans WATSON (A. E.), ALESSA (L.), SPRoull (J.) (eds), Wilderness in the Circumpolar North: searching for compatibility in ecological, traditional, and ecotourism values, U.S. Department of Agriculture, Forest Service, Rocky Mountain Research Station, Report RMRS-P-26, p. 43-53.

8. DYCKA (M. G.), BAYDACK (R. K.), 2004. - « Vigilance behaviour of polar bears (Ursus maritimus) in the context of wildlife-viewing activities at Churchill, Manitoba, Canada », Biological Conservation, $\mathrm{n}^{\circ} 116$, p. 343-350.

9. CROUZIER (P.), 2004. - Développement touristique et protection de l'environnement en milieux polaires: exemples du Svalbard et du Groenland, mémoire de Maîtrise, sous la direction de Samuel Étienne, Université de Clermont-Ferrand, Département de Géographie, 145 p.

10. Chauveau (M.), 2004 - Les valeurs écotouristiques de l'Islande, mémoire de Maîtrise, sous la direction de Samuel Étienne, Université de Clermont-Ferrand, Institut Universitaire Professionnalisé de Tourisme, $215 \mathrm{p}$. 
11. ERASO (A.), DOMÍNGUEZ (C.), 2004. - Glaciar caves and karst in polar regions, Proceedings of the 6th International symposium held on 3-8 septembre 2003 in Ny-Ålesund, Svalbard, Lat. $79^{\circ} \mathrm{N}$, Madrid, SEDECK, $193 \mathrm{p}$.

12. chevnina (E. V.), MedKova (O. N.), 2004. - «Fresh water investigation in Grenfjord area (Svalbard) », dans ERASO (A.), DOMÍNGUEZ (C.), Ibidem, p. 21-26.

13. MAVluYdov (B. R.), SOlovyanova (I. Y.), 2004. - «Drainage system of Aldegonda glacier, Spitsbergen ", dans ERASO (A.), ibid., p. 163-169.

14. GUDE (M.), 2000. - Ereignissequenzen und Sedimenttransporte im fluvialen Milieu kleiner Einzugsgebiete auf Spitzbergen, Heidelberger Geographische Arbeiten, Heft 110, 124 p.

15. HORMES (A.), 2004. - «Holocene glacier recessions on Svalbard inferred from radiocarbon dates of mosses found in en-glacial melwaterchannels », dans ERASO (A.), DOMÍNGUEZ (C.), Glaciar caves and karst in polar regions, op. cit., p. 145.

16. Polar Research, 2003, volume $22, \mathrm{n}^{\circ} 2$.

17. MACHERET (Y. Y.), MOSKALEVSKY (M. Y.), 2004. - « Study of outlet Lange Glacier, king George Island ice cap, South Shetland islands, Antarctica », dans ERASO (A.), DOMÍNGUEZ (C.), ibidem, p. 155.

18. ERASO (A.), PULINA (M.), 2001. - Cuevas en hielo y ríos bajo los glaciares, segunda edicíon, Serie MacGraw-Hill de divulgación científica, Madrid, McGraw Hill, 279 p.

19. Jouventin (P.), 2001. - « Les îles australes, laboratoires de l'évolution », Pour la Science, $\mathrm{n}^{\circ} 285$, p. 32-35.

20. fRenot (Y.), chapuis (J.-L.), Lebouvier (M.), 2001. - «La biodiversité dans les îles subantarctiques ", Pour la Science, $\mathrm{n}^{\circ} 285$, p. 36-41.

21. FRENOT (Y.) et al., 2001. - «Human activities, ecosystem disturbances and plant invasions in subantarctic Crozet, Kerguelen and Amsterdam Islands », Biological Conservation, vol. 101, p. 33-50.

22. JOUVEnTIN (Р.), 2001. - Les confessions d'un primate - les coulisses d'une recherche sur le comportement animal, Paris, Belin - Pour la Science, $189 \mathrm{p}$.

23. sonjal (S.), zalar (P.), plemenitas (A.), Cimerman (N. G.), « Fungal life in glacier ? », dans eraso (A.), DOMÍNGUEZ (C.), Glaciar caves and karst in polar regions, op. cit., p. 175-179.

24. PETIT (J. R.) et al., 1999. - « Climate and atmospheric history of the past 420,000 years from the Vostok ice core, Antarctica », Nature, vol. 399, p. 429-436.

25. Jouzel (J.), 2003. - «Climat du passé (400000 ans): des temps géologiques à la dérive actuelle », CRAS Geoscience, $\mathrm{n}^{\circ} 335$, p. 509-524.

26. EPICA Community Members (56 auteurs), 2004. - « Eight glacial cycles from an Antarctic ice core ", Nature, vol. 429, p. 623-628.

27. WALKeR (G.), 2004. - « Frozen time », Nature, vol. 429, p. 596-597.

28. MCMANUS (J. F.), 2004. - « A great grand-daddy of ice cores », Nature, vol. 429, p. 611- 612.

29. Berger (A.), LOUtRe (M. F.), dans DROXLER (A. W.), POORE (R. Z.), BURCKLE (L. H.), 2003. - « Earth's climate and orbital eccentricity: the marine isotope stage 11 Question », Geophys. Monogr., 137, Washington, AGU, p. 147-156.

30. IPCC, 2001. - IPCC Third Assessment Report: Climate Change 2001: The scientific basis, Cambridge, Cambridge University Press.

31. Cf. «Chronique Arctique », Norois, 1997, t. 44, n 175, p. 515-548.

32. NorthGRIP Members, 2004. - «High-resolution record of Northern Hemisphere climate extending into the last interglacial period », Nature, vol. 431, p. 147-151.

33. Cuffey (K. M.), 2004. - « Into an ice age », Nature, vol. 431, p. 133-134.

34. PAVLov (P.), SVENDSEN (J. I.), INDRELID (S.), 2001. - « Human presence in the European Arctic nearly 40,000 years ago ", Nature, vol. 413, p. 64-67.

35. GoWlett (J. A. J.), 2001. - « Out in the cold », Nature, vol. 413, p. 33-34. 
36. D'ARCANGUes (M.), 2002. - Dictionnaire des explorateurs des pôles, Paris, Séguier, 693 p.

37. BERG (K.), 2003. - Heroes of the polar wastes. Pioneer Norwegian explorers in the Arctic and the Antarctic, Andresen \& Butenschøn AS, 175 p.

38. Adrien de Gerlache de Gomery est le pionnier d'une grande famille d'explorateurs polaires belges à qui Charles $\mathrm{E}$. Schelfhout a consacré un magnifique ouvrage de 448 pages publié aux éditions de la Dyle et intitulé: Les Gerlache.Trois générations d'explorateurs polaires (1897-1997).

39. ABRUzzes (Duc des), 2004. - Expédition de l'étoile polaire dans la mer arctique 1899-1900, Paris, Economica, $288 \mathrm{p}$.

40. Dans la même collection Polaires à noter la publication en 2002 de trois ouvrages : Giddings (J. L.), 2002. - 10000 ans d'histoire arctique, Paris Economica, 496 p.; NOBILE (U.), 2002. - Le Pôle, aventure de ma vie, Paris Economica, 460 p. ; MALAURIE (J.), 2002. - De la vérité en ethnologie..., Paris, Economica, $408 \mathrm{p}$.

41. Parmi les beaux livres des éditions La Martinière publiés récemment, notons celui consacré à notre domaine : MALAURIE (J.), 2001. - L'appel du Nord, Paris, La Martinière, 352 p.

42. KNUTSEN (N. M.) et POSTI (P.), 2002. - La Recherche En ekspedisjon mot nord - Une expédition vers le Nord, Tromsø, Angelica Forlag AS, $240 \mathrm{p}$.

43. D’AUnEt (L.), 1992. - Voyage d'une femme au Spitzberg, Paris, Félin, 248 p.

44. HUSSENET (E.), 2004. - Rêveurs de Pôles. Les régions polaires dans l'imaginaire occidental, Paris, Septième Continent/Seuil, $192 \mathrm{p}$.

45. HuSSENET (E.), 2003. - Spitzberg. Visions d'un baladin des glaces, Paris, Transboréal, 2e édition, 128 p. ; HUSSENET (E.), 1998. - Maelström, Seul aux confins du Spitzberg, Paris, Transboréal, 333 p.

46. PRZYBYTAK (R.), 2003 - Variability of air temperature and atmospheric precipitation in the Arctic, Atmospheric and oceanographic sciences library, vol. 25, 352 p.; PRZYBYTAK (R.), 2003 - The climate of the Arctic, Atmospheric and oceanographic sciences library, vol. 26, 288 p. ; Bobylev (L. P.), KONDRATYEv (K. Y.), JOHANNESSEN (O. M.), 2003. - Arctic Environment Variability in the Context of Global Change, Springer Praxis Books, 471 p.; KIMBLE (J.M.) (éd.), 2004. - Cryosols: permafrost-affected soils, Springer, $726 \mathrm{p}$.

47. BAMBER (J.), PAYNe (A.), 2004. - Mass Balance of the Cryosphere. Observation and modelage of contemporary and future changes, Cambridge University Press, $662 \mathrm{p}$.

48. Dowdeswell (J.), HAMBRey (M.), 2002. - Islands of the Arctic, Cambridge University Press, 280 p.

49. RÉMY (F.), 2003. - L'Antarctique - la mémoire de la Terre vue de l'espace, Paris, CNRS, $180 \mathrm{p}$.

50. Bout (P.), CORbel (J.), DeRruAu (M.), GARAVEl (L.), PÉGUY (Ch.-P.), 1955. - « Géomorphologie et glaciologie en Islande centrale », Norois, $\mathrm{n}^{\circ} 8, \mathrm{p} .461-574$.

51. DECAUlne (A.), 2001. - Dynamique des versants et risques naturels dans les fjords d'Islande du nordouest, l'impact géomorphologique et humain des avalanches et des debris flows, Thèse de l'Université Blaise-Pascal, Clermont-Ferrand, 391 p. ; Étienne (S.), 2001. - Les processus de météorisation des surfaces basaltiques en Islande. Approche épistémologique de la géomorphologie des milieux froids, Thèse de l'Université de Paris I Panthéon-Sorbonne, $477 \mathrm{p}$.

52. ÉTIENNE (S.), 2004. - Islande. Biogéomorphologie d'un milieu périglaciaire humide, ClermontFerrand, Éditions Mélanie Séteun, 257 p.

53. ANDRÉ (M.-F.), Mercier (D.) (éd.), 2003. - « La recherche française actuelle dans les milieux polaires et subpolaires ", Bulletin de l'Association de Géographes Français, 4, p. 343-416.

54. Inter-Nord, $\mathrm{n}^{\circ}$ 20, 2003, $372 \mathrm{p}$.

55. «Imaginaires du Grand Nord ", Chemins d'étoiles, $n^{\circ}$ 10, 2003, Paris, Transboréal, 205 p.

56. ONFRAY (M.), 2002. - Esthétique du Pôle Nord, Stèles hyperboréennes, Paris, Grasset, 187 p.

57. DARRIEUSSECQ (M.), 2003. - White, Paris, POL, 222 p.

58. ECHENOZ (J.), 1999. - Je m'en vais, Paris, Minuit, 256 p., édité au format poche en 2001.

59. BENCHLEY (R.), 2002. - L'expédition polaire à bicyclette, Paris, Le Dilettante, 95 p. 


\section{AUTEURS}

\section{SAMUEL ÉTIENNE}

GEOLAB - UMR 6042 CNRS, Université Blaise Pascal Clermont-Ferrand, 4 rue Ledru - 63057

Clermont-Ferrand cedex, France,

setienne@seteun.net

\section{DENIS MERCIER}

EA 2579-DEPAM (UMR 6042 CNRS), Université Paris-Sorbonne (Paris IV), 1 rue Victor Cousin - 75 230 Paris cedex, France,

denis.mercier@paris4.sorbonne.fr

\section{MARIE-FRANÇOISE ANDRÉ}

GEOLAB - UMR 6042 CNRS, Université Blaise Pascal Clermont-Ferrand, 4 rue Ledru - 63057

Clermont-Ferrand cedex, France,

m-francoise.andre@univ-bpclermont.fr 